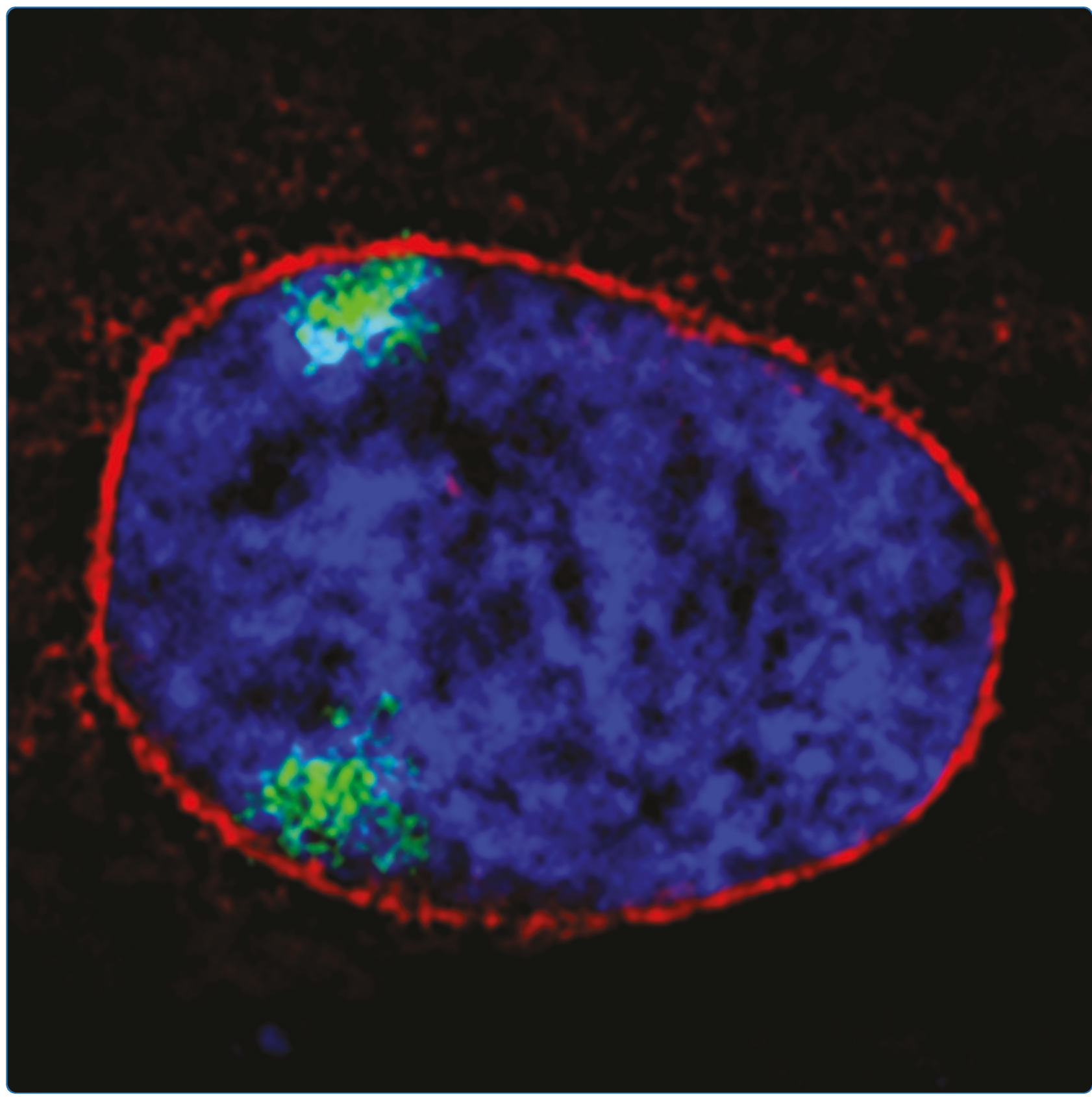

Specific nuclear envelope transmembrane proteins can promote the location of chromosomes to and from the nuclear periphery

Zuleger et al. 


\title{
Specific nuclear envelope transmembrane proteins can promote the location of chromosomes to and from the nuclear periphery
}

\author{
Nikolaj Zuleger ${ }^{1}$, Shelagh Boyle², David A Kelly ${ }^{1}$, Jose I de las Heras ${ }^{1}$, Vassiliki Lazou', Nadia Korfali ${ }^{1}$, \\ Dzmitry G Batrakou', K Natalie Randles ${ }^{3,4}$, Glenn E Morris ${ }^{3,4}$, David J Harrison ${ }^{5}$, Wendy A Bickmore ${ }^{2}$ and \\ Eric C Schirmer ${ }^{1 *}$
}

\begin{abstract}
Background: Different cell types have distinctive patterns of chromosome positioning in the nucleus. Although ectopic affinity-tethering of specific loci can be used to relocate chromosomes to the nuclear periphery, endogenous nuclear envelope proteins that control such a mechanism in mammalian cells have yet to be widely identified.

Results: To search for such proteins, 23 nuclear envelope transmembrane proteins were screened for their ability to promote peripheral localization of human chromosomes in HT1080 fibroblasts. Five of these proteins had strong effects on chromosome 5, but individual proteins affected different subsets of chromosomes. The repositioning effects were reversible and the proteins with effects all exhibited highly tissue-restricted patterns of expression. Depletion of two nuclear envelope transmembrane proteins that were preferentially expressed in liver each reduced the normal peripheral positioning of chromosome 5 in liver cells.
\end{abstract}

Conclusions: The discovery of nuclear envelope transmembrane proteins that can modulate chromosome position and have restricted patterns of expression may enable dissection of the functional relevance of tissue-specific patterns of radial chromosome positioning.

\section{Background}

It is well established that specific chromosomes, chromosome regions, and/or chromatin domains have preferred positions in the nucleus. For example, gene-poor chromosomes/regions tend to be at the nuclear periphery while gene-rich regions locate to the interior [1-5]. This radial organization can be modulated by the physiological and pathological state of the cell [6-8]. Genome organization can also be tissue specific [9-11]. For example, mouse chromosome 5 tends to be in the nuclear interior in liver but at the periphery in lung [12]. More dramatically, the radial organization of chromatin completely inverts in the nuclei of rod photoreceptors in nocturnal mammals [13]. In addition to visual methods, molecular approaches also reveal changes in associations of the

\footnotetext{
* Correspondence: e.schirmer@ed.ac.uk

'The Wellcome Trust Centre for Cell Biology, University of Edinburgh,

Mayfield Road, Edinburgh, EH9 3JR, UK

Full list of author information is available at the end of the article
}

mammalian genome with the nuclear lamins upon differentiation [14].

One might easily postulate that peripheral localization would depend on connections to the nuclear envelope (NE). Indeed, many NE proteins have chromatin binding activities important for reforming the NE at the end of mitosis $[15,16]$ and a high-affinity interaction established at this time can direct interphase chromosome positioning [17-19]. However, most NE-chromatin interactions described involve widely expressed proteins (reviewed in [20]) mediating interactions with heterochromatin [21-25]. Such interactions provide a mechanism to maintain inactive chromatin at the periphery that could explain the partial relationship between chromosome positioning and gene density. However, they do not explain how in some tissues particular chromosomes reposition to the nuclear periphery. Some work has been done indicating components that may be involved from the chromatin side, but the only proteins implicated from 
the NE thus far are lamins. However, though lamin B1 contributes to retention of specific chromosomes at the nuclear periphery, its ubiquitous presence is inconsistent with it directing tissue-specific chromosome positioning without additional factors [26,27]. That lamins support this process is further indicated by mutant forms of lamin A resulting in altered chromosome positioning in human nuclei $[8,28]$.

To identify other NE factors, 22 novel NE transmembrane proteins (NETs) [29] and emerin were screened for their ability to contribute to chromosome positioning patterns in human cells. Five NETs (NET5, NET29, NET39, NET45 and NET47) promoted repositioning of chromosome 5 to the periphery, but only two of these (NET29 and NET39) did so for chromosome 13. These NETs exhibited restricted tissue expression and several were preferentially expressed in liver with, for example, NET45 and NET47 expressed roughly 20 -fold higher in liver than in kidney. Correspondingly, chromosome 5 was more peripheral in the nuclei of human liver than in kidney. Chromosome repositioning effects of NETs were reversible; most notably, depletion of the two most liverspecific NETs in liver cells reduced the normal peripheral positioning of chromosome 5 . Thus, we postulate that these NETs may play a role in generating the more particular patterns of chromosome organization observed in certain tissues.

\section{Results}

\section{A screen for NETs that influence chromosome positioning}

To identify NE proteins that contribute to genome organization, 22 novel NETs identified in a proteomic study of liver NEs [29] and the well-characterized NET emerin were screened to determine if they could influence the position relative to the nuclear periphery of lacO arrays integrated in two different human chromosomes. The inner nuclear membrane localization of most of the NETs tested here has been confirmed by super-resolution light microscopy and/or immuno-electron microscopy $[30,31]$. The lacO array was inserted into chromosome 5 (line 5.1) or 13 (line 2.7) of HT1080 human fibrosarcoma cells that stably express lac repressor (lacI) fused to green fluorescent protein (GFP) to visualize the array position. The lacO integration sites are known to faithfully reflect the nuclear organization of their counterpart human chromosomes [32].

Full-length NETs fused to monomeric red fluorescent protein (mRFP) were transiently expressed in line 5.1 (Figure 1a). This resulted in some accumulation of the fusion proteins in the endoplasmic reticulum (presumably due to saturation of binding sites at the NE), but a clear rim at the nuclear periphery was also observed (Figure 1b). Western blots for the mRFP tag revealed that the expressed NETs were not degraded (data not shown).
The lacO array in line 5.1 is randomly distributed and tends to not be at the periphery [32] as can be observed in the untransfected cells in each image. While this minimal peripheral positioning was unchanged in cells expressing most NETs (for example, NET55 in Figure 1b), the array was often observed at the periphery in cells expressing certain NETs (for example, NET29 and NET39 in Figure $1 \mathrm{~b})$.

The position of the array with respect to the nuclear periphery was quantified in two dimensions to avoid errors from the unequal resolution between $x y$ and $z$ inherent to light microscopy. By using only cells where the lacO array could be visualized at the midplane (the focus point at which the nuclear diameter is at its widest; Figure 1c) such errors in $\mathrm{z}$ could be eliminated. As this would be expected to remove from consideration arrays at the top or bottom of the nucleus, it is likely to slightly underestimate array movement to the periphery. A script was then employed that erodes the total nuclear area, as defined by DAPI staining, into five concentric shells of equal area from the outside (shell 1) to the inside (shell 5) [3] (Figure 1c). The incidence of the array in each shell was quantified for each NET from at least 50 cells and the percentage of the total incidence in each sector of the nucleus plotted.

The line 5.1 lacO array is randomly distributed (not enriched at the periphery), with roughly $20 \%$ in each of the five shells, and expression of mRFP alone did not change this compared to untransfected cells (Figure 1d). Expression of NET5 (TMEM201), NET29 (TMEM120A), NET39 (PPAPDC3), NET45 (DAK) and NET47 (TM7SF2) all increased the incidence of the lacO array at the nuclear periphery with high statistical significance of $P<0.01$ using $\chi^{2}$ tests to compare the incidence of the array in the peripheral shell (shell 1) against the combined internal shells (shells 3,4 and 5) between the NET-expressing cells and the mRFP-expressing cells. In contrast, emerin and most of the tested NETs had no strong significant effects on the position of the array, though NET23, NET33, NET38, and NET54 had minor effects using a lower stringency threshold of $P<0.05$. Throughout the text high stringency (double asterisks in figures) indicates $P$-values $<0.01$ while low stringency (single asterisks in figures) indicates $P$-values $<0.05$; all $P$-values are given in Additional file 1.

As expected for an integration into the gene-poor chromosome 13 [2], the lacO array in line 2.7 tends to be close to the nuclear periphery (shell 1) in approximately $50 \%$ of cells (Figure 2a). NET29 and NET39 further increased this to approximately $60 \%$ (Figure $2 b$ ). Thus, their effect was qualitatively the same for both integration sites studied. In contrast, NET47, which had strongly promoted peripheral incidence for the array in line 5.1 (Figure 1d), did not further promote peripheral incidence 

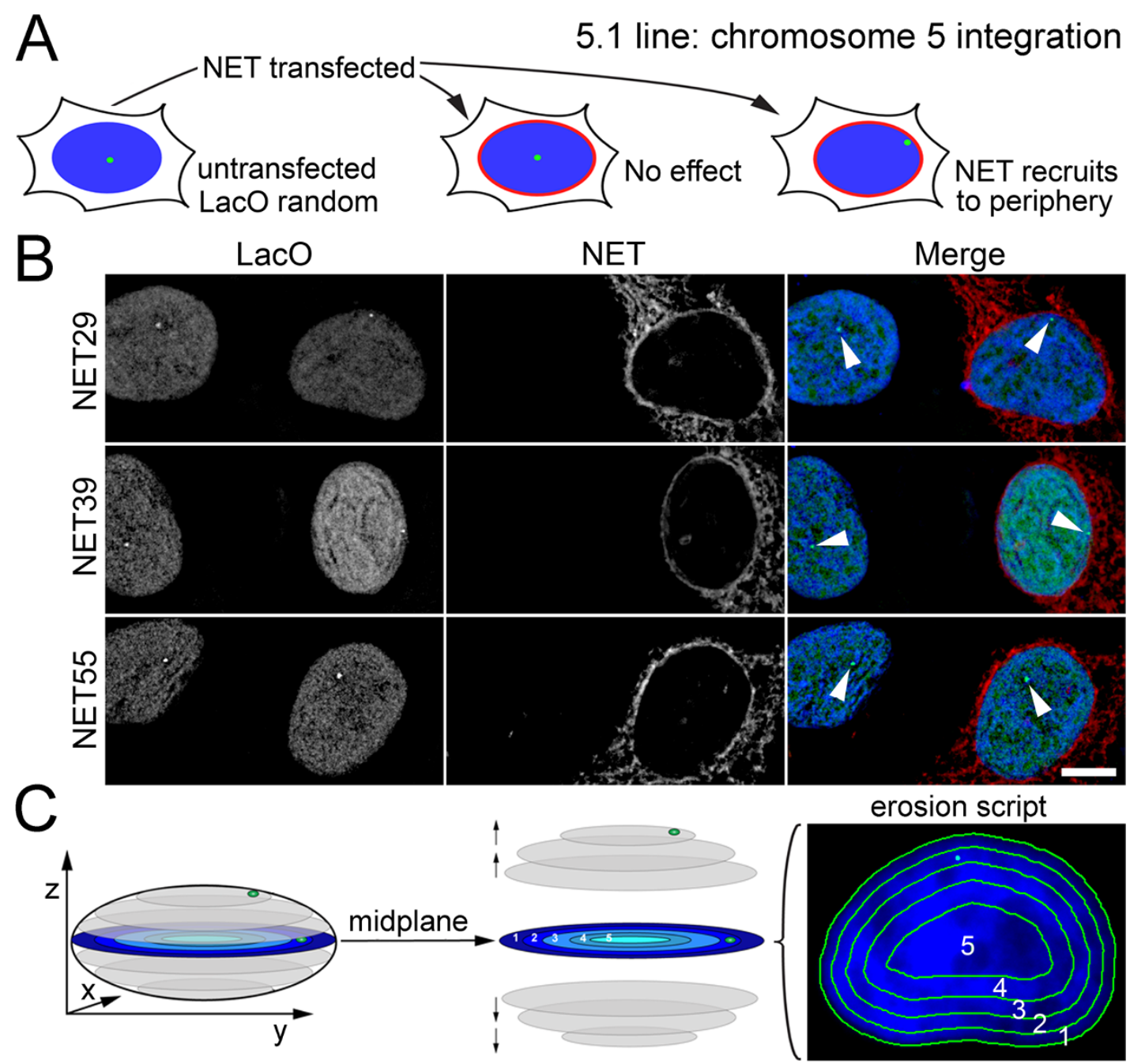

\section{erosion script}

D 5.1 line: chromosome 5 integration

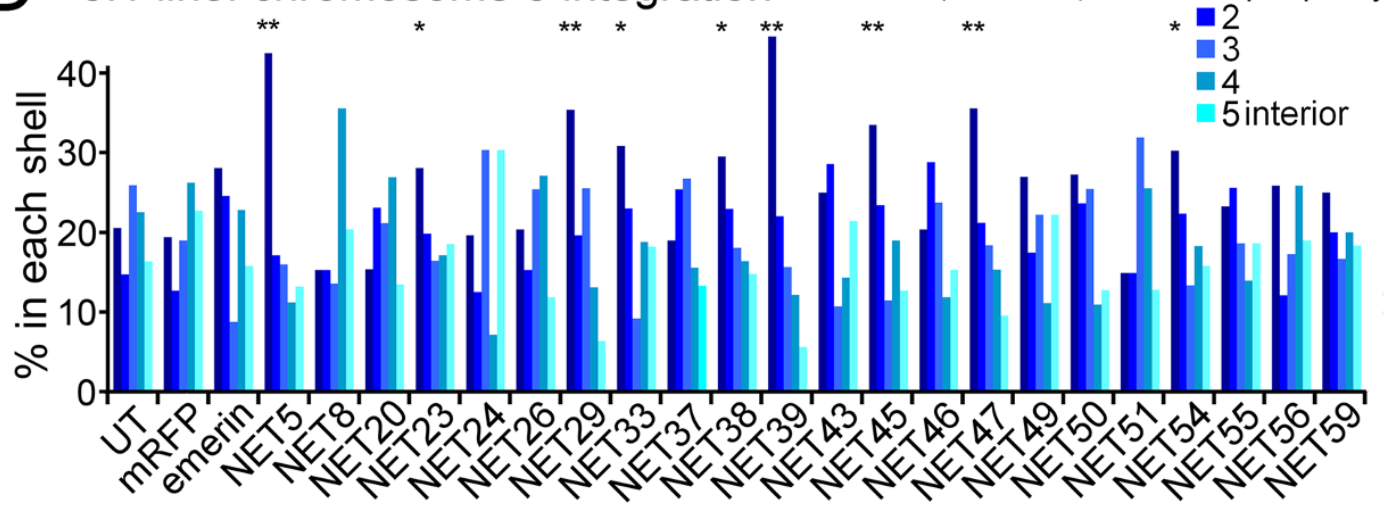

Figure $1 \mathrm{~A}$ screen to identify NETs that recruit chromosomes to the nuclear periphery. (a) An integrated lacO array marks chromosome 5 that tends to not be at the periphery in line 5.1. NETs fused to mRFP were exogenously expressed to screen for those involved in tethering chromatin to the NE. (b) Detection of the lacO array (arrowheads) and the transfected NETs (red) in the 5.1 line. The lacO array was not observed at the periphery in the untransfected cells (left) or the NET55 transfected cells, but was observed at the periphery in NET29 and NET39 transfected cells. Scale bar, $5 \mu \mathrm{m}$. (c) lacO array position was determined relative to five shells of equal area eroded from the periphery (1) to the centre (5) of the nucleus. To avoid errors in z from lacO arrays at the top or bottom of the nucleus, cells were only analyzed if the array occurred in the midplane where arrays occurring in the inner shells would be clearly internal. (d) Percentage of lacO-tagged loci in each of five erosion shells is plotted with the nuclear periphery (shell 1) to the left in dark blue and more internal localization occurring to the right and with lightening shades of blue. $n=50$ cells for each NET. Single asterisks designate NETs with lower stringency $P$-values $<0.05$ comparing the position of the array in the NET-transfected cells to the mRFP control using $\chi^{2}$ tests; double asterisks designate NETs with higher stringency $P$-values $<0.01$. Statistics for all NETs are given in Additional file 1. UT, untransfected. 


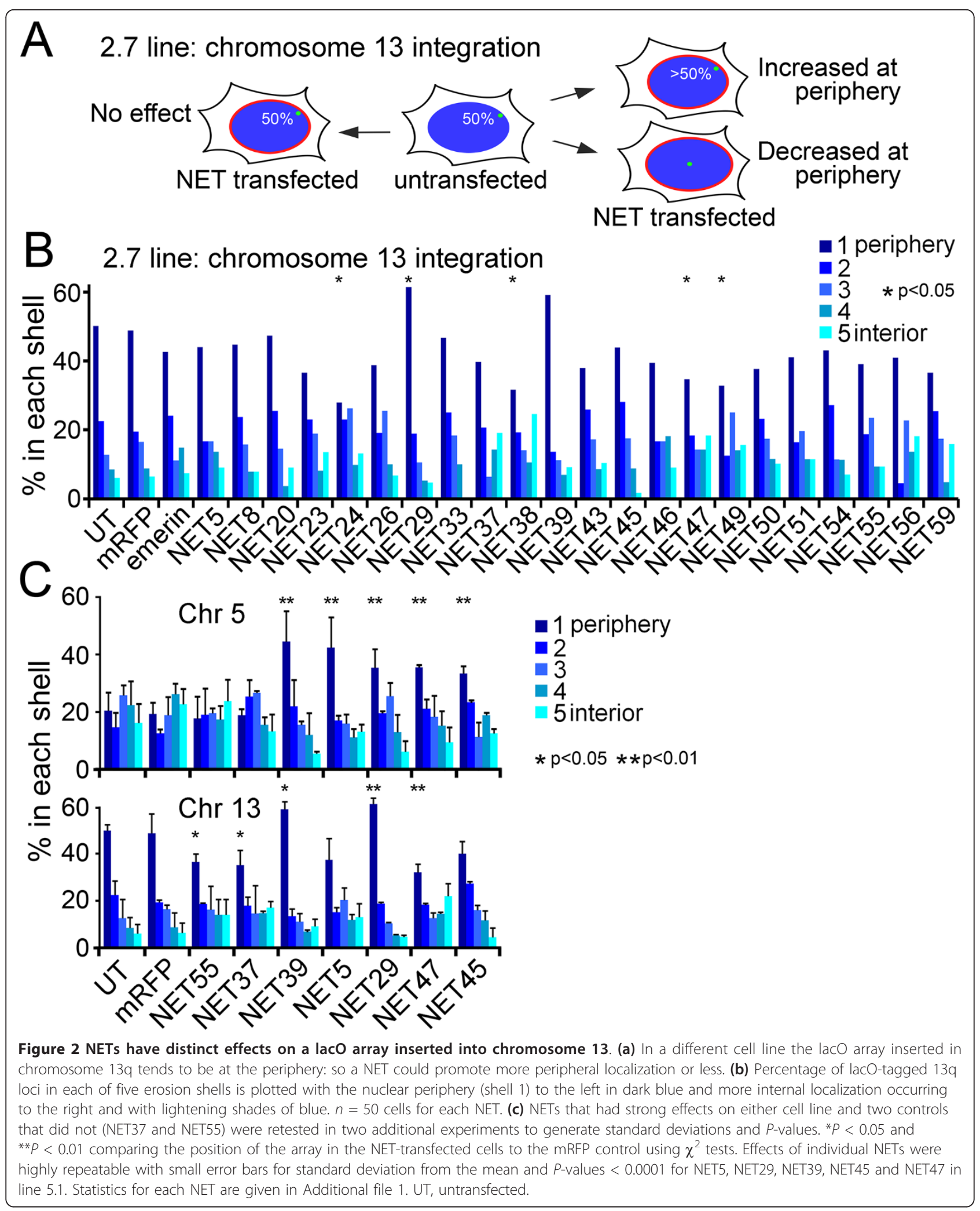


of the array in line 2.7 (Figure $2 \mathrm{~b}$ ). In fact, in cells expressing NET47 the incidence of the array at the periphery was less than in the untransfected and mRFP controls.

The five NETs that most strongly influenced array position in line 5.1 (NET5, NET29, NET39, NET45 and NET47), the untransfected (UT) and mRFP controls, and two NETs that had no effect (NET37 and NET55) were subjected to additional rounds of transfection in both lines to confirm the effects and increase the statistical sample size (Figure 2c). All three experiments were highly reproducible, with NET29 and NET39 strongly promoting peripheral positioning in both lines with very small error bars for the standard deviation of the mean, while NET47 promoted peripheral positioning in line 5.1 but not in line 2.7. In line 5.1 analyzing the combined datasets further increased the statistical significance, with $\chi^{2}$ tests yielding $P$-values of $<0.00001$ for all NETs with effects on chromosome position when comparing the NETs to the mRFP-transfected controls (Additional file 1). In line $2.7 \chi^{2}$ tests revealed high statistical significance for NET2 2 and low statistical significance for NET39, with both increasing peripheral incidence of the array. In contrast, NET5 and NET45, though increasing peripheral incidence of the 5.1 line, had no significant effects on the 2.7 line. Interestingly, the reduced incidence of chromosome 13 at the periphery in cells expressing NET47 was found to be highly significant with a $P$-value of 0.002 .

Gross changes in nuclear size and shape could in theory alter spatial genome organization [33], enabling more chromosomes to accumulate at the periphery. If a NET altered these parameters, it could have an indirect effect on chromosome positioning. Thus, nuclear size was determined by measuring the midplane area and nuclear shape was evaluated by measuring the longest and shortest cross-section in HT1080 cell nuclei expressing the NETs that affected chromosome position. Nuclear shape and size were largely unaffected by all these NETs (Figure 3a). The nucleolus could potentially also influence chromosome position but staining for nucleolin revealed no changes in the general appearance and number of nucleoli in cells transfected for the NETs that affected chromosome position (Figure $3 \mathrm{~b}$ ). The positioning effects were likely not due to disruption or redistribution of other NE proteins because lamin $\mathrm{A} / \mathrm{C}$, NPC proteins and the NETs emerin, SUN2 and nesprin 2 exhibited normal targeting in cells transfected with the NETs that affected chromosome position (Figure $3 \mathrm{~b}$ and data not shown). The integrity of NPC function and the NE as a permeability barrier were also tested by investigating the distribution of a reporter transport cargo in cells co-transfected with the NETs that affected chromosome position. The transport cargo concentrated in the nucleoplasm in all NET-transfected cells while transfection with a positive control (ICP27) that has been shown to interfere with transport [34] caused the reporter cargo to accumulate in the cytoplasm (Figure 3c).

\section{NETs reposition specific chromosomes}

To confirm that NET-induced repositioning of the lacO array faithfully represented the repositioning of the whole host chromosome, transfected 5.1 cells were subjected to fluorescence in situ hybridization (FISH) for the lacO array and for a chromosome 5 paint simultaneously. In the NET37-expressing control both the lacO array and the majority of chromosome 5 were internal whereas upon expression of NET39 the lacO array, the copy of chromosome 5 carrying the integrated lacO array, and the wild-type chromosome 5 were closer to the nuclear periphery (Figure 4a). Moreover, the lacO array itself was not directly at the periphery while other parts of the chromosome in images appeared to be in tight association with the NE. Importantly, this indicates that the lacO array itself does not contribute to NET39mediated recruitment of chromosome 5 to the periphery.

To further test if chromosome repositioning effects are independent of the lacO array, parental HT1080 cells lacking the array were stably transfected with four NETs that had the strongest positioning effects in the first screen (NET5, NET29, NET39, and NET47) and controls (NLS[nuclear localization signal]-GFP, NET37 and NET55) that did not. FISH for chromosome 5 showed that the NETs that promoted peripheral repositioning of the lacO array integrated into chromosome 5 also repositioned the whole of chromosome 5 in parental HT1080 cells lacking the array (Figure 4b). NET37 and NET55 that failed to reposition the lacO array had no effect on chromosome 5 positioning in the parental HT1080 cells (Figure 4b).

The positioning effects were not due to a general increase in peripheral chromatin as no differences were observed in DAPI signal distribution within the shells between the mRFP control and NETs that recruited the array to the periphery (Figure 4c). Correspondingly, chromosome 19, which is normally located in the nuclear interior $[3,35]$ remained in the nuclear interior. Indeed, approximately $90 \%$ of the hybridization signal from chromosome 19 was internally located in wild-type HT1080 cells and this distribution was unchanged in cells expressing each of the NETs that repositioned chromosome 5 to the periphery (Figure $4 \mathrm{~d}$ ). Thus, the NETs do not change the total distribution of chromatin in the nucleus, but must exhibit some specificity in their effects on chromosomes.

To test for such specificity, suggested already by the different effects of some NETs for the two lacO lines, this investigation was extended to a larger set of chromosomes $(1,5,11,13$ and 17) for a few NETs using both 

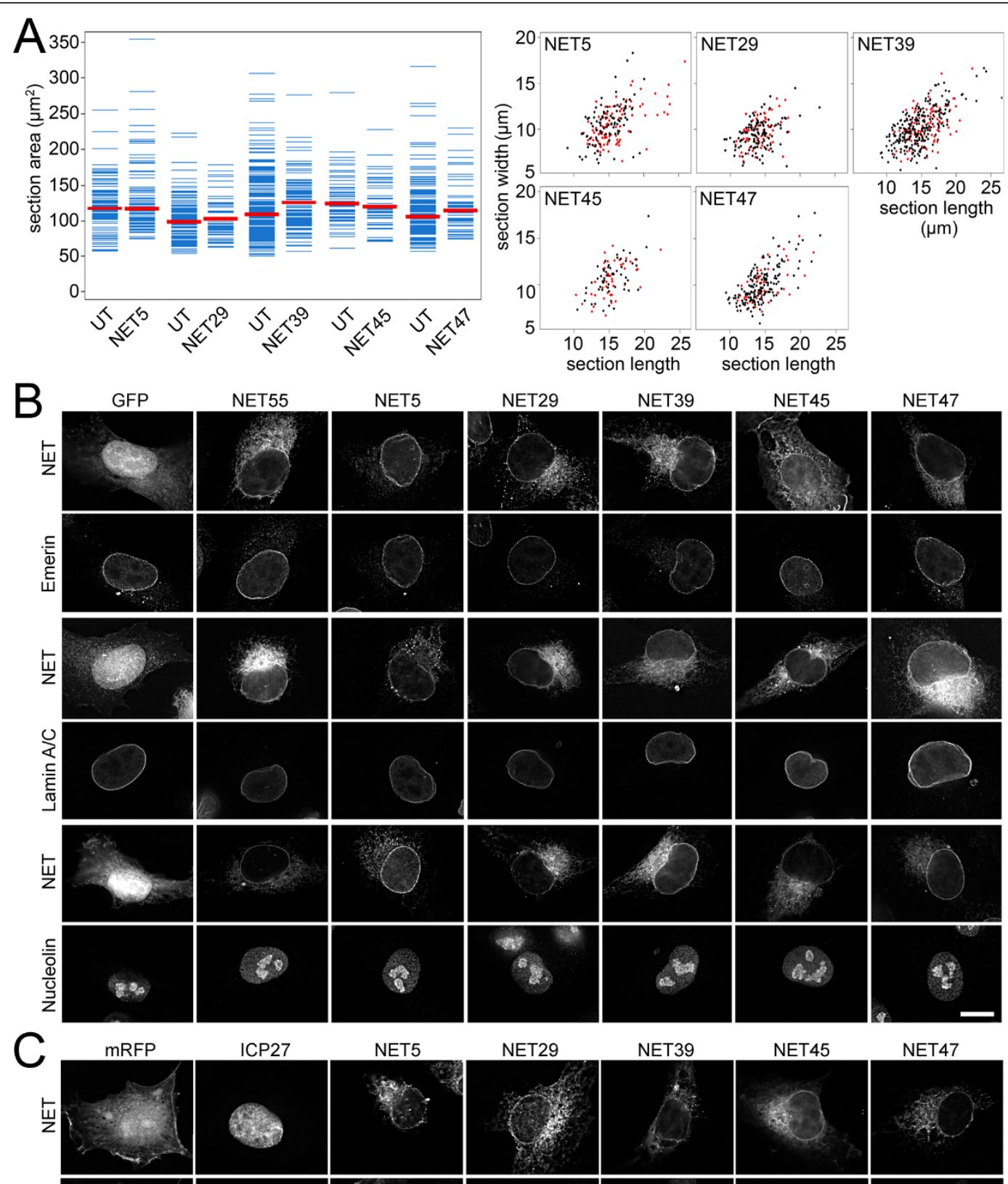

ICP27
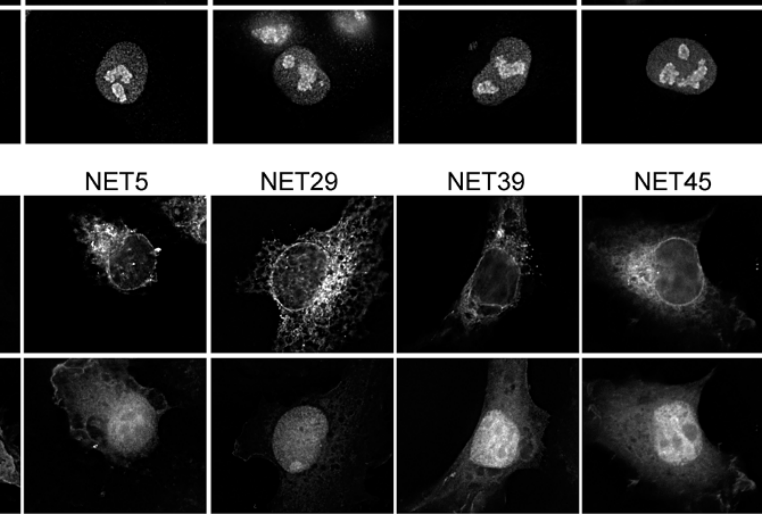

NET39
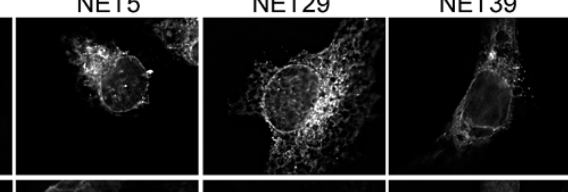

NET45

NET47
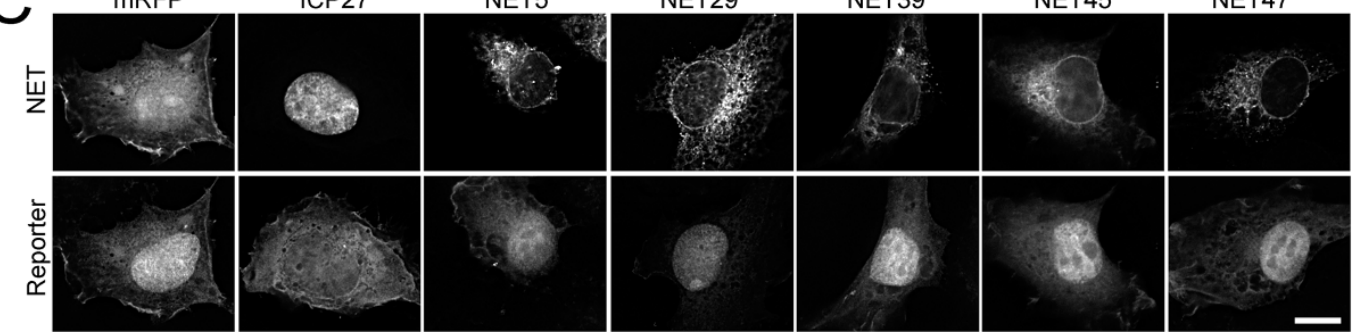

Figure 3 Positioning effects are not due to changes in nuclear size and shape, distribution of other nuclear proteins, or loss of nuclear permeability. HT1080 cells transiently transfected with tagged NETs were tested for parameters that could theoretically indirectly influence chromosome position. (a) The area of the nuclear midplane (left, distribution plots) and the longest and shortest distance across it (right, scatter plots) were measured and quantified for over 40 transfected cells for each NET. In each case the NET transfected cells were compared to untransfected (UT) cells in the same population. Only very moderate fluctuations in nuclear size or shape were observed. (b) Transfected cells were stained with antibodies for nucleolin to determine if NETs had indirect disruptive effects on nucleoli and for lamins and the NET emerin to determine if NETs generally altered NE organization. No changes in the distribution of these markers were observed. (c) The permeability barrier function of the NE in NET-transfected HT1080 cells was tested by expressing a reporter transport cargo in the same cells. The cargo contains two GFP molecules in tandem with a strong nuclear localization signal and a weak nuclear export signal so that it can shuttle but accumulates predominantly in the nucleus. If nucleocytoplasmic transport or NE permeability is compromised, the reporter accumulates more in the cytoplasm as when the herpesvirus protein ICP27, which interferes with nuclear transport through binding to Nup62, is co-expressed (second panel). None of the NETs affected the distribution of the reporter. For both (b) and (c) scale bar $=10 \mu \mathrm{m}$. 


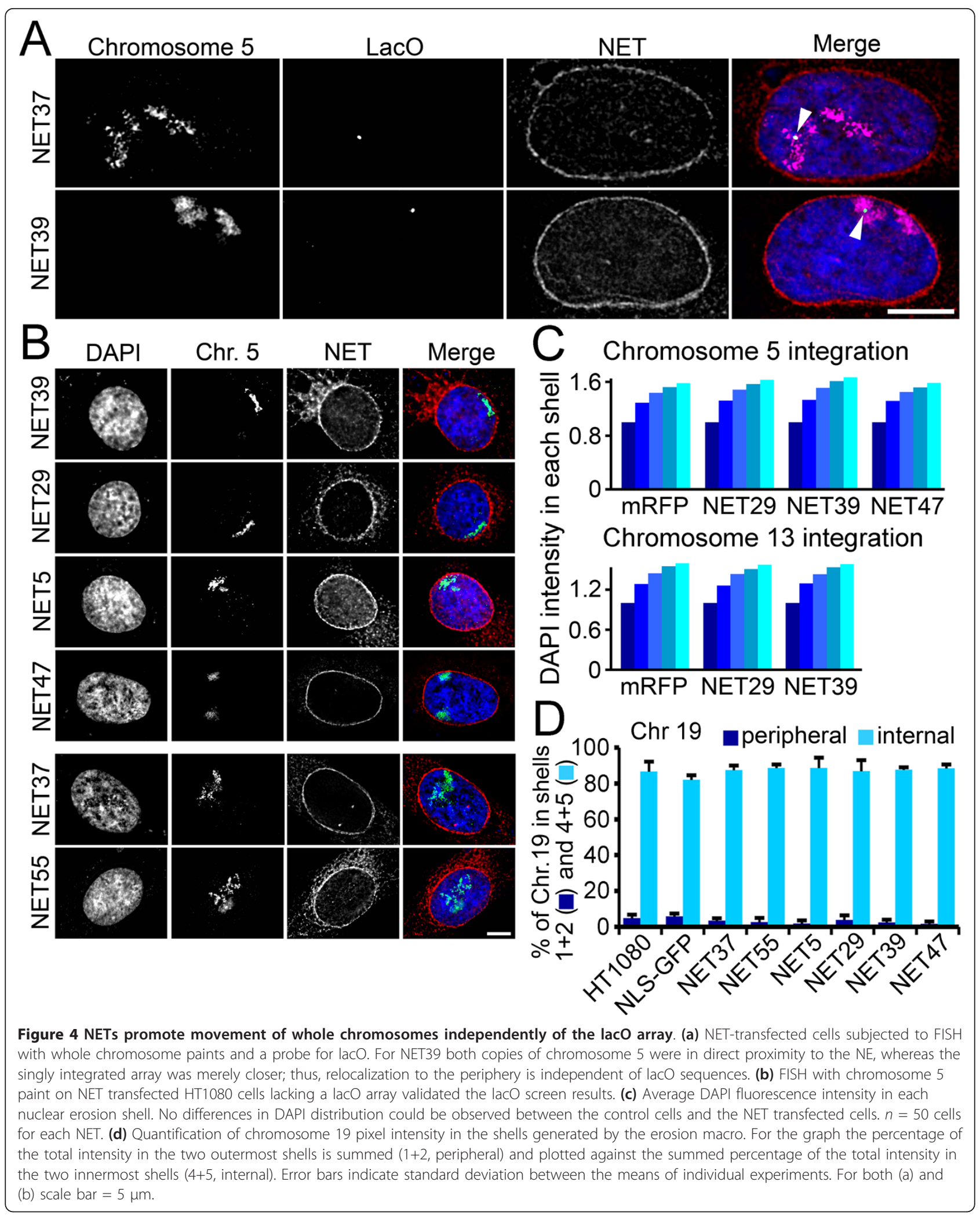


two-dimensional analysis of midplane images and threedimensional shell erosion analysis (analysis from threedimensional reconstructions). For the two-dimensional analysis the erosion shell macro was modified to measure the pixel intensity within each shell and the percentage of the total pixel intensity in the two most peripheral and two most internal shells was summed and plotted for 100 cells. For three-dimensional analysis a similar erosion script was applied to three-dimensional reconstructions from deconvolved stacks of images (Figure 5a). Because the large volumes for chromosome territories cross over several erosion shells, the unequal resolution in $\mathrm{z}$ should have little impact on three-dimensional measurements. For technical reasons the nucleus was divided into six concentric shells of equal area, rather than five as for the two-dimensional erosion analysis. As in the two-dimensional erosion analysis, the percentages of the total pixel intensity in the two outermost shells were combined and compared with the combined two innermost shells. As small regions of an internal chromosome can loop out to the periphery [36-38], the data are plotted as a percentage of the total pixel intensity so as to convey not just the movement of a chromosome region towards the periphery, but also how much of the whole chromosome moves towards the periphery. As the chromosome occupies multiple shells, statistical significance was assessed by comparing the distributions of chromosome intensities in the periphery (shells $1+2$ ) between the NLS-GFP control and the NET by means of the Kolmogorov-Smirnov (KS) test.

Both the two- and three-dimensional analyses show the same tendencies that were observed using the lacO array as a marker for chromosome position. NET5, NET29, NET39 and NET47 promoted chromosome 5 positioning to the periphery and NET29 and NET39 further increased the incidence of chromosome 13 at the periphery. The effects of NET29, NET39, and NET47 were even more significant when analyzing chromosome 5 than the line 5.1 array (KS test $P$-values for two-dimensional analysis of $2.4 \times 10^{-10}, 6.3 \times 10^{-11}$, and $4.1 \times 10^{-6}$, respectively), and despite the low number of cells in the three-dimensional analysis (approximately 20 ), all had significant $P$-values (Additional file 1). NET5 was also significant in both two-dimensional and threedimensional chromosome analysis, but went from highly significant with the lacO array to the lower significance cut-off of $P<0.05$. This difference in the strength of the effect for NET5 likely reflects a smaller portion of the chromosome at the periphery, consistent with the images shown in Figure 4b. Chromosome 11 appeared visually to be moderately repositioned to the nuclear periphery by expression of NET39 and NET47 in both twodimensional and three-dimensional analysis (Figure $5 \mathrm{~b}$ ); however, the $P$-values were only significant at the lower cut-off in the two-dimensional analysis. Chromosome 17, like chromosome 19 mentioned earlier, was unaffected by all NETs tested (Figure 5b; Additional file 1). Chromosome 1 did not increase in peripheral incidence with either NET, but instead appeared to exhibit a minor reduction in its peripheral incidence with both NETs. In all, NET39 promoted peripheral redistribution for three out of six chromosomes tested by the two-dimensional analysis while NET47 promoted peripheral redistribution for two of the chromosomes tested. Thus, NETs affect just a subset of chromosomes, with each NET promoting a particular pattern of chromosome repositioning.

\section{Chromosome repositioning effects of NETs are weakened without NE localization}

To test if chromosome repositioning likely requires a membrane anchor on the NET, HT1080 lines were generated that stably expressed just the soluble nucleoplasmic regions of NETs (Figure 6a) fused to GFP and an NLS. Topologies were experimentally determined (data not shown) from accessibility in digitonin-permeabilized cells [39]. NET5 was not tested because it has two separate large nucleoplasmic regions. The fusions to the soluble nucleoplasmic regions of NET29, NET 39 and NET47 all accumulated diffusely in the nucleoplasm (Figure 6b), and two had weak effects $(P<0.05)$. However, none had the strong effects on chromosome positioning as were seen for their full-length counterparts (Figure 6c; compare with Figure 5b).

\section{NET-directed chromosome repositioning is reversible}

Chromosome 13 tended to be peripheral in the HT1080 fibroblast cells and the peripheral incidence was increased with exogenous expression of NET29 or NET39. As the endogenous NET29 and NET39 proteins were also present in wild-type HT1080 cells, the tendency towards peripheral positioning for chromosome 13 could be due to this basal expression of the NETs. Thus, to test whether peripheral positioning of chromosomes by NETs is reversible, NET29 and NET39 were knocked down using RNA interference in wild-type HT1080 cells (Figure 7). Immunoblotting indicated that small interfering RNAs (siRNAs) reduced the protein expression levels to 30 to $50 \%$ of the normal endogenous levels by day 5 and 10 to $40 \%$ by day 7 (Figure 7a). Knockdown of each NET on its own was able to reduce the peripheral incidence of chromosome 13 by approximately 30\% (Figure 7b). Both were statistically significant at day 7 of the knockdown using the KS test comparing to the scramble control from the same day of the knockdown (Additional file 1). This further confirms the NET function in positioning chromosomes as well as indicating that multiple NETs can act on the same chromosome. 


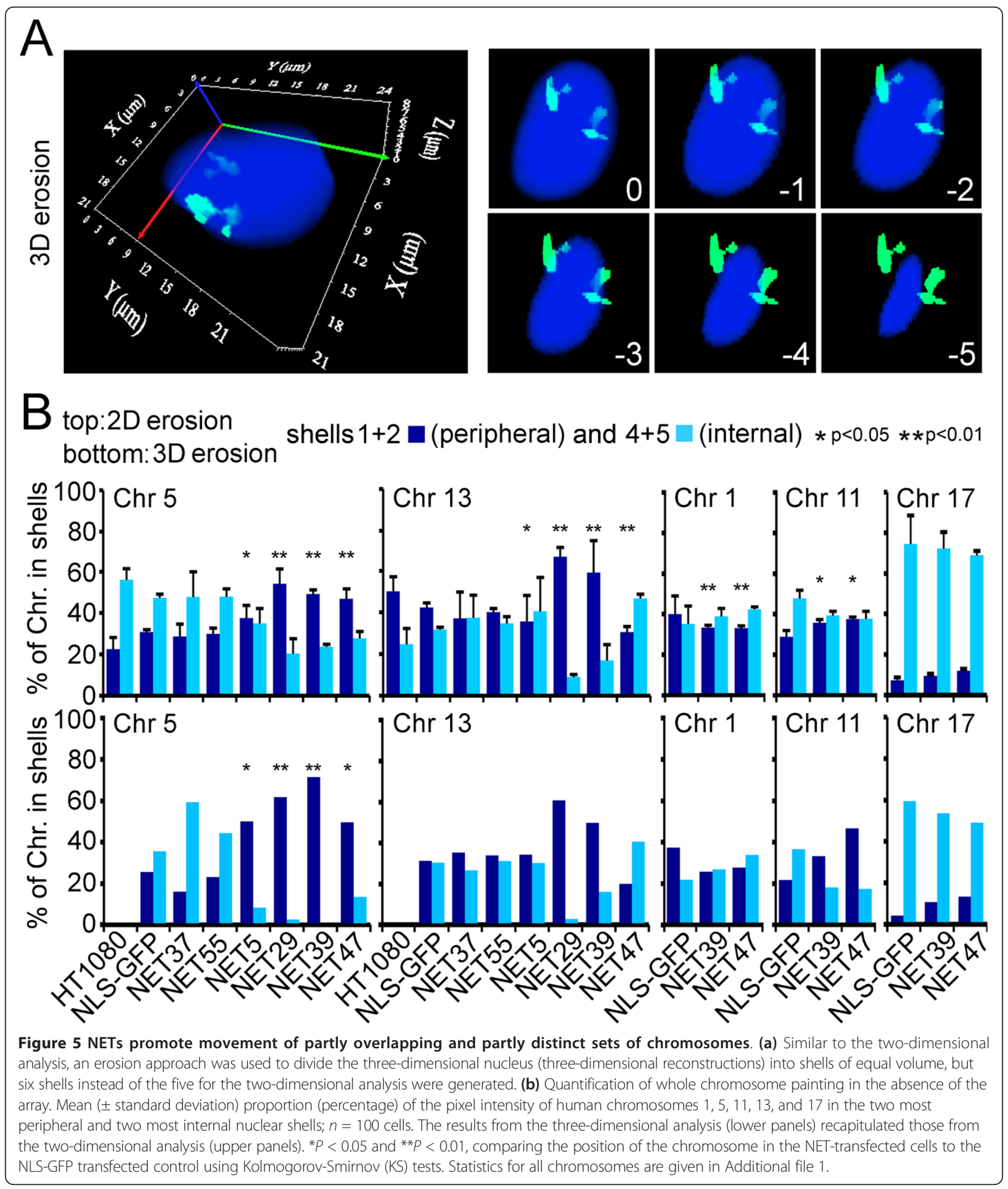

Restricted tissue expression of NETs that affect chromosome positioning

Given their effects on the positioning of certain chromosomes by both over-expressing and knocking down expression, these NETs could explain previous observations of tissue-specific chromosome positioning $[11,12,40,41]$ if they are restricted in their expression. Thus, the protein levels of NETs with effects in the chromosome-repositioning screen were determined in different human tissues by western blot. All NETs that 


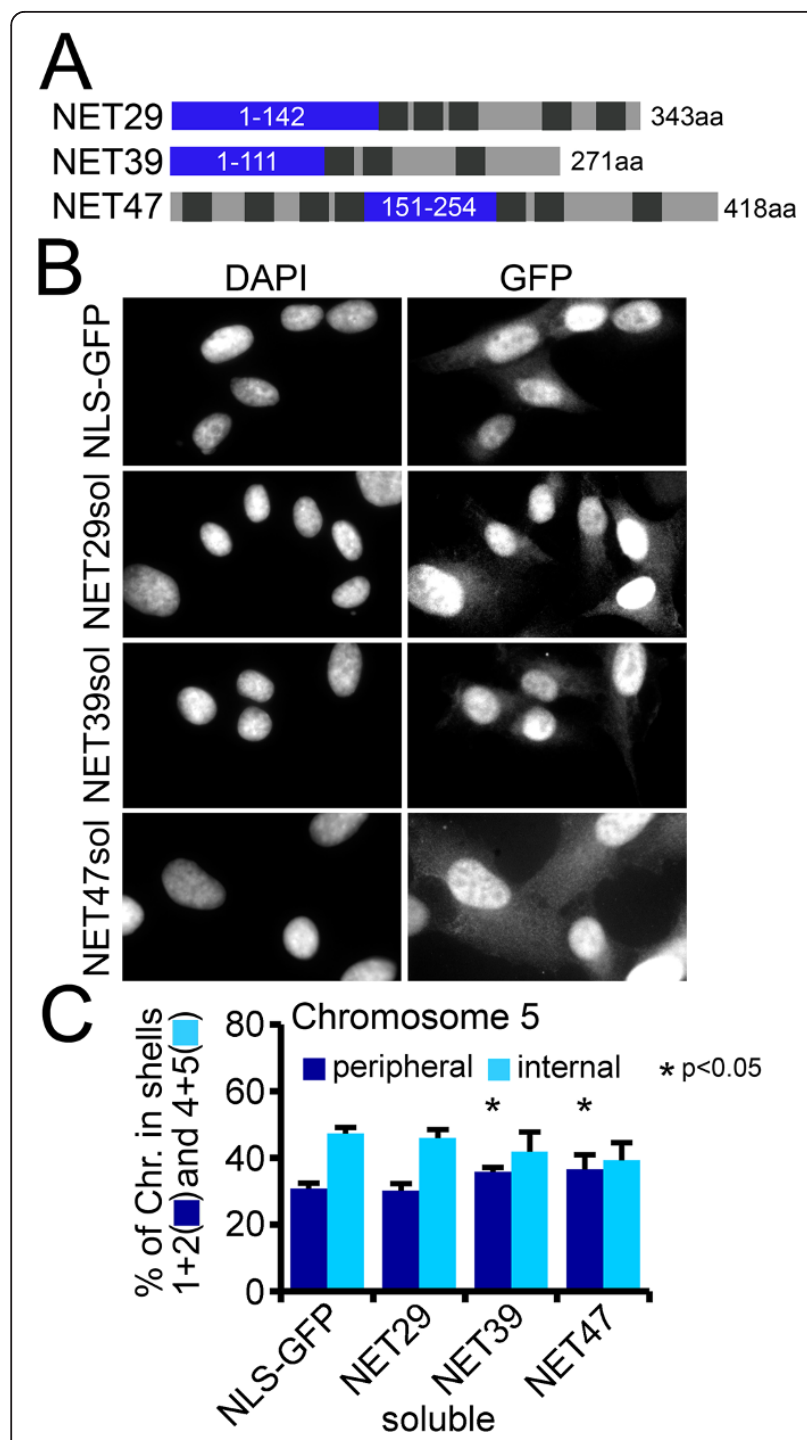

Figure 6 Removal of the transmembrane segments reduces effects of NETs in chromosome repositioning. Soluble fragments of NETs from their largest predicted nucleoplasmic segment were fused to an NLS and stably expressed in HT1080 cells to determine if they could influence chromosome repositioning. (a) Schematic diagram of each NET highlighting the soluble fragment in blue. The dark grey boxes indicate predicted transmembrane spans. (b) Images showing nucleoplasmic targeting of the GFP fusion constructs. (c) Cells expressing soluble fragments encoding the principal nucleoplasmic regions of NETs did not recapitulate the strong effects in repositioning observed with full-length NETs (Figure 5b). Error bars indicate standard deviation between the means of individual experiments. ${ }^{*} P<0.05$ comparing the position of the chromosome in the NET-transfected cells to the NLS-GFP transfected control using KS tests. None of the soluble NET fragments yielded higher stringency $P$-values $<0.01$. Statistics are given in Additional file 1 .

altered chromosome position were restricted in expression to a subset of tissues (Figure 8). As the NETs used in the initial screen were identified in a proteomic analysis of liver NEs [29] it is not surprising that
NET45 and NET47 are very preferentially and highly expressed in liver. NET5 was expressed at very low levels in liver, but more strongly in brain, muscle and testis. Notably, the presence of bands with distinct molecular weights in some tissues indicates that some NETs have either splice variants or major post-translational modifications that are tissue-specific. In contrast, NET55 and NET20, which did not have repositioning effects, were widely expressed (Figure 8). Similarly, other NE-related proteins, SUN2, lamin B2, Ran, and the loading control GAPDH, were widely expressed in all tissues (Figure 8).

The protein levels on the western blot were measured in each tissue, summed, and the percentage of the total signal across all tissues observed in each particular tissue was calculated (Additional file 2). Thus, if a protein were expressed evenly across all 11 tissues it would have $9 \%$ of the total signal in each tissue. NET20, which visibly was relatively evenly expressed across all tissues, ranged from a low of $5 \%$ in ovary to a high of $13 \%$ in liver. In contrast, $77 \%$ of the total NET 45 signal and $70 \%$ of the total NET47 signal was found in liver. Only $4 \%$ of the total NET45 signal and 3\% of the total NET47 signal was found in kidney, making the relative expression in liver roughly 20 -fold higher than in kidney for both NETs.

NET involvement in chromosome positions in liver cells The highly preferential expression of NET45 and NET47 in liver together with their roughly 20-fold lower expression in kidney led us to test the position of chromosome 5 in human liver and kidney sections. Chromosome 5 was more associated with the nuclear periphery in human liver while being more associated with the nuclear interior in kidney with significant $P$-values from KS tests comparing the two tissues (Figure 9a, b).

HepG2 hepatocellular carcinoma cells retain many characteristics of hepatocytes [42], which comprise roughly $80 \%$ of liver. As in human liver sections, chromosome 5 was also predominantly peripheral in the HepG2 nuclei. To test whether this is dependent on the highly preferentially expressed liver NETs, NET45 and NET47 were co-depleted using siRNAs in HepG2 cells and the position of chromosome 5 monitored by FISH (Figure 9c, d). By day 4 protein levels had been depleted to between 10 and $30 \%$ of normal levels (Figure 9c). The percentage of chromosome 5 hybridization signal at the nuclear periphery decreased over time after combined siRNA treatment for the two NETs, but not for the control scrambled siRNA, and the proportion in the nuclear interior correspondingly increased (Figure 9e). At 4 days of depletion the chromosome repositioning was significant $(P=0.0169)$ using KS tests comparing the knockdown to the scramble control of the same day, and this improved to $P=7.6 \times 10^{-5}$ by 7 days. Depletion of each NET alone reduced the peripheral localization of 


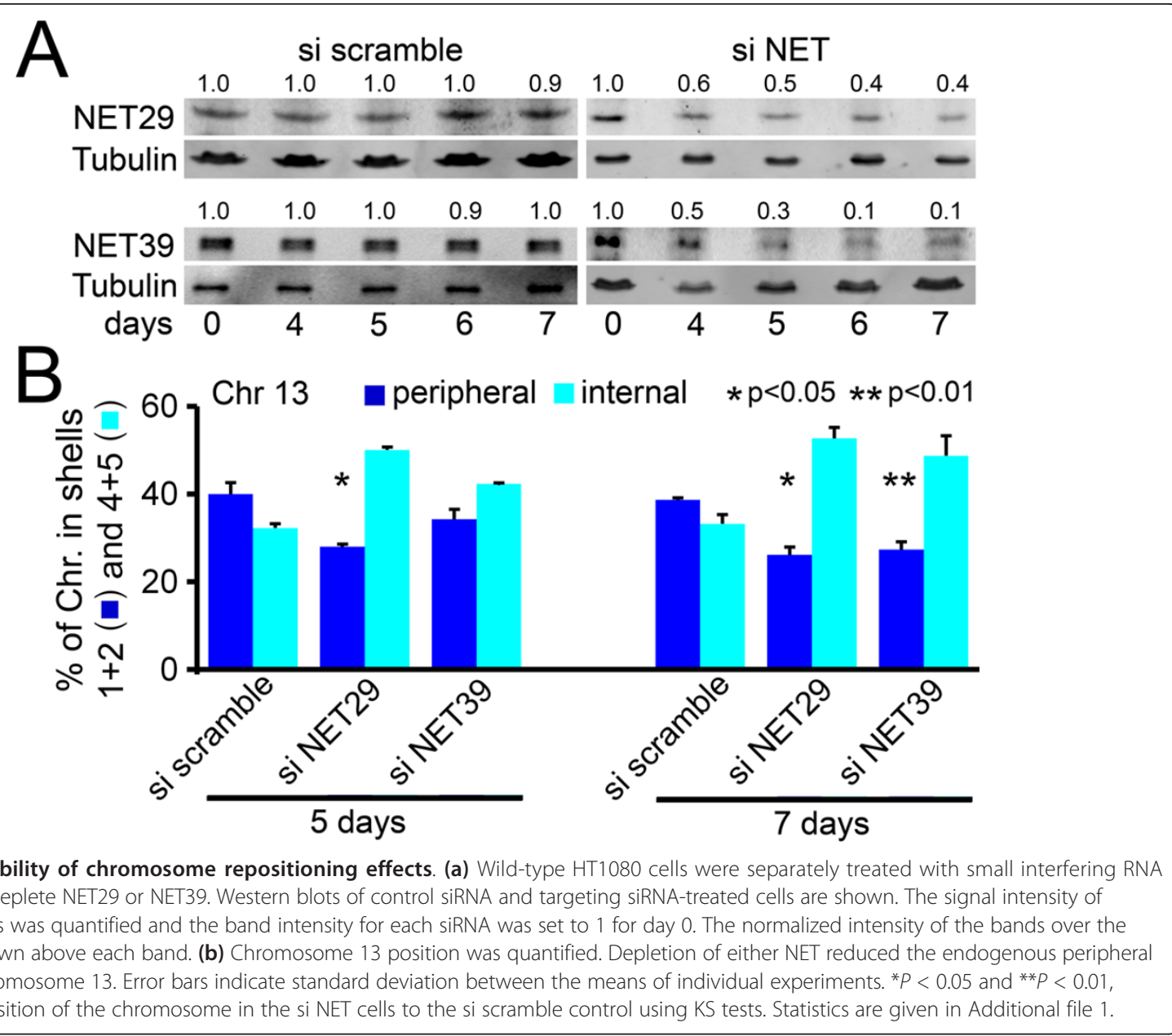

chromosome 5 with statistical significance, but depletion of both NETs together reduced peripheral localization to only a slightly greater extent than NET45 alone (Figure 9f; Additional file 1). Thus, though both NET45 and NET47 contribute to the tethering of chromosome 5 in liver, it is not clear whether they act together or independently of one another.

\section{Discussion}

We have identified nuclear membrane proteins that have extremely restricted patterns of expression in tissues and can promote particular patterns of spatial genome organization. Most NE-chromatin interactions described previously involved widely expressed proteins [20] mediating interactions with general heterochromatin [21-25,43]. Such interactions provide a mechanism for maintaining silent chromatin at the periphery that could help explain the partial relationship between chromosome positioning and gene density and indeed manipulating histone methylation can also affect heterochromatin positioning at the NE [44]. The release of chromosome 18 from the periphery due to lamin B1 defects [26] likely reflects such a mechanism because both lamin B1 and the core histones it binds [45-47] are ubiquitously expressed. The same should apply for the lamin A effects on chromosome position; however, lamin A mutations associated with disease affected gene and chromosome positioning in a more tissue-specific manner $[28,48,49]$. For example, lamin A mutations blocked release of a tissue-specific promoter from the periphery in the tissue matched to the promoter, but did not affect the peripheral localization in other tissues [48]. This suggests that lamin A is not the only protein involved in the initial tethering. Instead, we propose that lamins might reinforce a chromosome arrangement initially established by a tissue-restricted NET or be required for NE targeting of the NET, consistent with recent studies on lamin-associated domain organization by lamin B1 in conjunction with LAP2ß and HDAC3 [27] and generalized heterochromatin association with the periphery mediated by lamins in conjunction with the lamin B receptor (LBR) [43].

We postulate that the tissue-restricted NETs we have found function in similar complexes with lamins and chromatin proteins. There has been far more research 

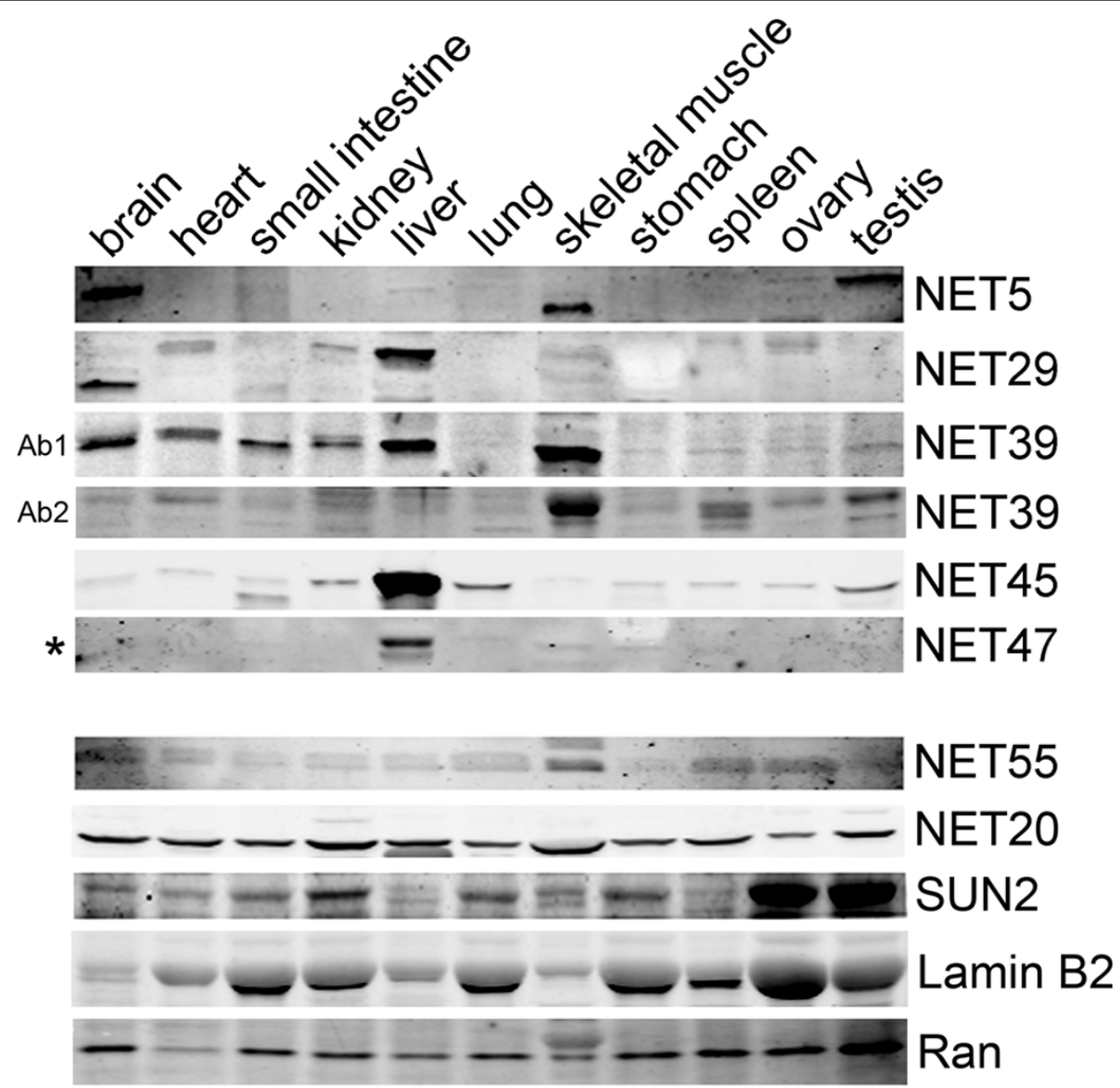

\section{GAPDH}

Figure $8 \mathrm{NETs}$ that recruit chromosomes to the nuclear periphery have restricted tissue expression. Human tissue blots equally loaded for the tissues listed (20 $\mathrm{\mu g}$ in each lane) were probed with NET antibodies. NETs that had strong chromosome repositioning effects tended to be highly restricted in their expression. In contrast, several other NE proteins and controls were expressed in all tissues (lower panels). Ab1 and Ab2: two commercial antibodies to NET39 revealed restricted tissue expression and the highest levels of expression in muscle, but differed in the reactivity with some other tissues. Asterisk: NET47 migrates at $38 \mathrm{kDa}$ instead of the calculated $46 \mathrm{kDa}$.

to identify proteins on chromatin involved in tissue-specific patterns of genome organization compared to what has been done to identify the NE component. For example, in Caenorhabditis. elegans, release from the periphery of a muscle-specific promoter could be effected by the muscle-specific transcriptional regulator HLH-1 [10]. This argues that tissue-specific genes on chromosomes and transcriptional complexes sitting on them may be the mechanism by which specificity of chromosome positioning is conferred on the chromosome side because these tissue-specific genes would be unevenly distributed on the chromosomes. Different gene regulatory complexes that interact with different NETs could in theory work together to achieve a threshold affinity for chromosome tethering. That both NET29 and
NET39 knockdown reduced the peripheral incidence of chromosome 13 in HT1080 cells and both liver-preferential NET47 and NET45 knockdown in the liver HepG2 cells reduced the peripheral incidence of chromosome 5 is consistent with this idea. However, much work still needs to be done to identify the specific binding partners on chromatin, to investigate the functions of these NETs in both matched and unmatched tissues, and test them in different combinations. Further, although the loss of chromosome repositioning function with the soluble fragments is consistent with the NETs being the physical tether at the NE, we cannot exclude the possibility that the NETs have a less direct function in modifying a different tether protein or chromatin component. Furthermore, although NET5, NET29, 

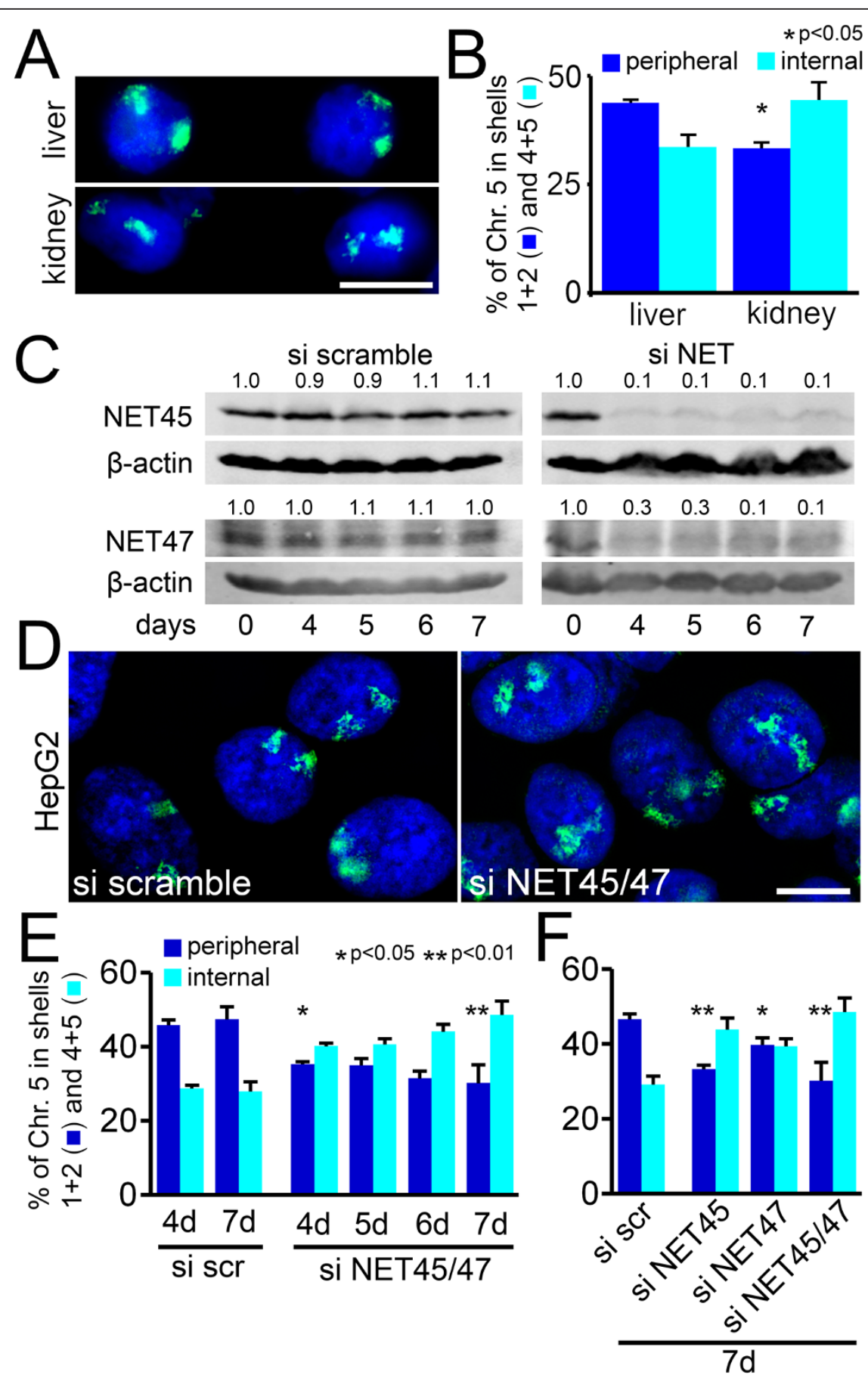

Figure 9 Peripheral chromosome 5 position in liver is reduced by depletion of liver NETs. (a) Chromosome 5 in human liver or kidney sections. Scale bar, 5 m. (b) Chromosome 5 positioning was quantified for the two tissues $(n=100)$. (c) NET45 and NET47 were depleted by RNA interference in HepG2 cells, a liver-derived human cell line. A scrambled siRNA sequence was used as a control. The knockdown over time is shown by western blot. The signal intensity of bands on the blots was quantified and the band intensity for each siRNA was set to 1 for day 0 . The normalized intensity of the bands over the time course is shown above each band. (d) Chromosome 5 in siRNA-treated HepG2 cells (left, scramble control; right, NET depleted). Scale bar, $5 \mu \mathrm{m}$. (e) Quantification of the results in (d); 100 cells were counted for each timepoint. The percentage of chromosomes relocated away from the periphery by the combined NET45/NET47 knockdown increased over time, also strengthening the statistical significance. (f) NET45 and NET47 were also tested individually to determine if both were needed for the normal peripheral localization. Each alone reduced the percentage of chromosome 5 at the periphery. The combined knockdown had a stronger effect than either NET alone $(n=100)$. Error bars indicate standard deviation between the means of individual experiments. ${ }^{*} P<0.05$ and ${ }^{* *} P<0.01$, comparing the position of the chromosome in the si NET cells to the si scramble control using KS tests. Statistics are given in Additional file 1. 
NET39 and NET47 were all resistant to a pre-fixation detergent extraction characteristic of lamina-associated NETs and characterized at the inner nuclear membrane by super resolution microscopy and in some cases immuno-electron microscopy [29-31], the fact that with overexpression much accumulates in the endoplasmic reticulum raises the possibility that some effects could be mediated indirectly from the cytoplasm.

The partial reduction in peripheral positioning observed for chromosome 13 in cells expressing NET47 could imply an indirect effect for these NETs in modifying a signaling pathway that can go in either direction, that is, movement towards the periphery or movement away from the periphery. However, it is also perfectly consistent with a model where different NETs recruiting partially overlapping and partially distinct sets of chromosomes to the periphery could indirectly cause the release of a particular chromosome from the periphery by increasing the peripheral tethering of a different chromosome.

Of the NETs that had effects in this screen, NET29, NET39, NET45 and NET47 are uncharacterized for any chromatin-related functions and there are no shared sequence characteristics among them. Only NET5 (also known as Tmem201 and Samp1) has been experimentally indicated to be able to bind to DNA or chromatin [50,51]. NET47 (also known as TM7SF2 and delta(14)-sterol reductase), which has a separate enzymatic function [52-54], may also be able to bind chromatin because it shares considerable sequence similarity with LBR $[52,55]$ that binds heterochromatin protein 1 (HP1) [56]. Part of this HP1 binding site has been mapped close to the first transmembrane domain of LBR [57], a region that has high homology with NET47. Additionally, LBR has also been shown to be involved in spatial olfactory receptor gene organization [58]. Interestingly, both NET39 and NET47 have been implicated in tissue-specific processes, with NET39 playing a role in myogenesis $[59,60]$ and NET47 knockout mice exhibiting a reduction in gene expression from a number of liver-specific genes [61].

Although there is still much work to do in testing whether these tissue-restricted NETs are tissue-specific in their functions and identifying the interaction partners/ sites on chromosomes, the effects of NET47 on liverspecific gene expression [61] suggests that future work with these NETs will also answer questions on the functional consequences of particular patterns of spatial genome organization. Many aspects of the effects of spatial genome organization on gene expression remain contentious. For example, three studies tethering a lacO array to the NE yielded three different effects on gene expression [17-19]. Future studies manipulating tissue-restricted NETs in matched tissue systems may be the key to clearly answering many questions about the functional consequences of spatial genome organization.

\section{Conclusions}

Certain chromosomes exhibit tissue-specific patterns of radial positioning in nuclei and several studies have demonstrated that chromosome tethering to the nuclear envelope can be achieved through an affinity mechanism. However, the endogenous proteins responsible for establishing a particular pattern of radial chromosome positioning have remained elusive. Here we identify several tissue-restricted nuclear envelope transmembrane proteins that can alter the radial position of chromosomes in the nucleus. Exogenous expression of each of these proteins in a general fibroblast cell line can recruit particular sets of chromosomes to the nuclear periphery and the peripheral distribution of certain chromosomes in differentiated cells can be reduced by depletion of these proteins. We predict that these newly discovered tissue-restricted NETs will likely play a pivotal role in dissecting the functional relevance of tissue-specific patterns of radial chromosome positioning in development.

\section{Materials and methods \\ Plasmid construction}

IMAGE clones for human NETs were inserted into a monomeric red fluorescent protein vector (pmRFP) as described [30]. Those used for stable cell lines were moved to the clontech pEGFP-N2 vector except for NET39, which was moved to PEGFP-C1 via EcoRI/BamHI sites. Soluble fragments are schematized in Figure 6. The reporter plasmid $\operatorname{Rev}_{48-116}-\mathrm{GFP}_{2}$-cNLS (NES-GFP ${ }_{2}$-cNLS) was described in [62].

\section{Cell culture and transfections}

LacO cell lines were generated previously from HT1080 fibrosarcoma cells in the Bickmore laboratory [32]. Human HT1080 cells and these derivatives (lines 5.1, 2.7, and those stably expressing NETs) and human HepG2 hepatocellular carcinoma cells were maintained in high glucose DMEM supplemented with $10 \%$ fetal bovine serum, $100 \mu \mathrm{g} / \mathrm{ml}$ penicillin and $100 \mu \mathrm{g} / \mathrm{ml}$ streptomycin sulfate. Cells were plated at approximately $10 \%$ confluency to prevent their reaching confluency before fixation at $72 \mathrm{~h}$ post-transfection. DNA was transfected $12 \mathrm{~h}$ after plating using Fugene 6 (Promega, Madison, WI, USA) according to the manufacturer's instructions.

HT1080 cells were stably transfected using linearized plasmids carrying NET-GFP fusions. Transfectants were initially selected for with $500 \mu \mathrm{g} / \mathrm{ml}$ Geneticin for 2 weeks and surviving cells were further enriched for those expressing the GFP fusions by fluorescence-activated cell 
sorting (FACS). Cells were maintained thereafter with $100 \mu \mathrm{g} / \mathrm{ml}$ Geneticin.

\section{Antibodies and tissue western blots}

Human tissue blots (IMB-103, IMGENEX, San Diego, CA, USA) were probed using standard procedures with antibodies to NETs. Antibodies against NET5 (06-1013), NET29 (06-1018), NET55 (06-1029), SUN2 (06-1038) were all rabbit polyclonals generated to peptides from Millipore (Temecula, CA, USA). Antibodies to NET39 were rabbit polyclonals from either Millipore (06-1025; Ab1) or Proteintech (20635-1-AP [Chicago, IL, USA]; Ab2). Antibodies to NET47 were rabbit polyclonals from either Millipore (06-1026) or Professor Rita Roberti, Perugia University [53]. NET45, mouse monoclonal antibodies were generated against a recombinant GST-NET45 fusion protein lacking transmembrane domains using methods previously described [63]. NET20 antibodies were rabbit polyclonals made to peptide KFKRNLSVEAEVDLLSYCAR (amino acids 83 to 102). Lamin B2 rabbit polyclonals have been previously described (3932) [64]. Ran mouse monoclonal and GAPDH rabbit polyclonal antibodies were from BecktonDickenson (610341, San Jose, CA, USA) and EnoGene (E1C604, New York, NY, USA), respectively. Protein bands were visualized with IR680- or IR800-conjugated secondary antibodies using a LI-COR Odyssey (LI-COR, Lincoln, NE, USA). Quantification of bands in western blots was performed using the LI-COR Odyssey Application Software version 3.0. Integrated intensities were calculated from an equal size box drawn around each band in each tissue (Table S2a in Additional file 2). To assess the variation of protein abundance among tissues, the intensities were summed within each protein for all tissues, and then expressed as a percentage of the signal per tissue (Table S2b in Additional file 2).

For other western blots, protein lysates from knockdown experiments were separated on SDS-PAGE and transferred to membranes that were probed with the primary antibodies listed above and as loading controls anti-actin (mouse monoclonal; A1978, Sigma, St. Louis, MO, USA) or anti-tubulin (mouse monoclonal; T6074, Sigma). Because GFP is denatured during FISH procedures, to visualize GFP-NETs antibody A11122 (Life Technologies, Grand Island, NY, USA) against GFP was used. Antibodies against lamin A/C (rabbit polyclonal 3262) [64], emerin (MANEM1 5D10) [65], nucleolin (rabbit polyclonal Ab22758, Abcam, Cambridge, UK) were also used to stain the NET-transfected cells in Figure 3.

\section{Immunofluorescence staining and permeability assays}

Transiently transfected cells were fixed in $4 \%$ paraformaldehyde for 7 minutes, then permeabilized in $0.2 \%$ Triton X-100/PBS for 5 minutes and blocked with $2 \%$
BSA in PBS. They were then incubated with primary antibodies at $37^{\circ} \mathrm{C}$ for $1 \mathrm{~h}$ in the same $2 \%$ BSA solution, washed three times in PBS and then incubated with secondary antibodies for 45 minutes. After washing in PBS, cells were mounted in fluoromount G (EM Sciences, Hatfield, PA, USA) and analyzed by microscopy.

For permeability assays HT1080 cells were transiently transfected with tagged NETs fused to MRFP and the transport reporter NES-GFP ${ }_{2}$-cNLS [62], which has both nuclear export and import signals so that it shuttles, but the import signal is dominant. Thus, the reporter should accumulate predominantly in the nucleoplasm unless transport or NE permeability is compromised. As a positive control for disruption of transport, cells were cotransfected with the reporter and pmRFP-C1 plasmid encoding WT ICP27. After $24 \mathrm{~h}$ cells were fixed and processed for microscopy.

\section{siRNA knockdown of NETs}

siRNA oligos were used for knockdown of NET29 (5'CUA AGU UUG CCU ACA AGG A[dT][dT]-3' and 5'UCC UUG UAG GCA AAC UUA G[dT][dT]-3') and NET39 (5'- CUA CCU CAC CAU GGA CAU CUA[dT] [dT]-3' and 5'- UAG AUG UCC AUG GUG AGG UAG [dT][dT]-3') in HT1080 cells; $8 \mu \mathrm{g}$ of either was transfected into $10^{6}$ cells using a Nucleofector (Lonza, Cologne, Germany) with program L-005 and solution T. The control siRNA was a scrambled sequence. Knockdown was confirmed using the Millipore NET29 and Proteintech NET39 antibodies.

For knockdown of NET45 and NET47 in HepG2 cells, $3 \mu \mathrm{g}$ each of Smartpools (Thermo Fisher Scientific, Waltham, MA, USA: NET45 L-006808-00-0020, NET47 L-005744-00-0020) alone or in combination were transfected into $1.5 \times 10^{6}$ cells using nucleofection (Lonza, solution V, program T-028). The control siRNA was a scrambled sequence. Knockdown was confirmed using the monoclonal NET45 antibody and the NET47 antibody from Professor Rita Roberti.

\section{Fluorescence in situ hybridization}

For immuno-FISH, cells were fixed in $4 \%$ paraformaldehyde, aged 2 days, then permeabilized in $0.2 \%$ Triton $\mathrm{X}-100 / \mathrm{PBS}$ for 5 minutes and incubated with primary antibodies at $37^{\circ} \mathrm{C}$ for $1 \mathrm{~h}$ and secondary antibodies for 45 minutes. After washing in PBS, cells were again fixed with $2 \%$ formaldehyde for 5 minutes to fix antibodies prior to denaturing FISH steps. Cells were permeabilized again with $0.5 \%$ Triton X-100, washed in PBS and then pre-equilibrated in $2 \times$ SSC and treated with RNase (100 $\mu \mathrm{g} / \mathrm{ml}$ ) at $37^{\circ} \mathrm{C}$ for $1 \mathrm{~h}$. After washing in $2 \times \mathrm{SSC}$, cells were dehydrated with a $70 \%, 90 \%$ and $100 \%$ ethanol series. Slides were heated at $70^{\circ} \mathrm{C}$ and then submerged in pre-heated $\left(80^{\circ} \mathrm{C}\right) 70 \%$ formamide $/ 2 \times \mathrm{SSC}(\mathrm{pH} 7.2)$ for 
20 minutes followed by another ethanol dehydration series. Slides were air-dried and hybridized to biotinlabeled chromosome paints (CamBio, Cambridge, UK) or a lacO probe labeled with digoxigenin. Hybridizations were incubated for 2 days at $37^{\circ} \mathrm{C}$, then washed in $2 \times$ SSC at $45^{\circ} \mathrm{C}$ followed by $0.1 \times \mathrm{SSC}$ at $60^{\circ} \mathrm{C}$. Slides were then pre-equilibrated in $4 \times$ SSC, $0.1 \%$ Tween- 20 and blocked with BSA for 15 minutes before incubating with avidin or digoxigenin antibodies (Roche). DNA was visualized with DAPI (4,6-diamidino-2 phenylindole, dihydrochloride) and coverslips mounted in fluoromount G (EM Sciences, Hatfield, PA, USA).

Human tissue sections were obtained according to local ethics protocols, formalin fixed, paraffin embedded and sectioned at 5 to $6 \mu \mathrm{m}$ thickness onto slides. Sections were subsequently prepared for whole chromosome painting according to published protocols [66]. Briefly, sections were deparaffinized in $100 \%$ xylene at $45^{\circ} \mathrm{C}$, rehydrated with an ethanol series, permeabilized with $1 \mathrm{M} \mathrm{Na}$ isothiocyanate at $80^{\circ} \mathrm{C}$ for 30 minutes followed by $50 \mu \mathrm{g} / \mathrm{ml}$ pepsin (Sigma P-6887) in $0.01 \mathrm{~N} \mathrm{HCl}$ at $37^{\circ}$ $\mathrm{C}$ for 45 minutes. Cells were then dehydrated with an ethanol series and whole chromosome painting was performed as above.

\section{Microscopy}

Most images were obtained using a Nikon TE-2000 microscope equipped with a 1.45 NA $100 \times$ objective, Sedat quad filter set, PIFOC Z-axis focus drive (Physik Instruments, Cranfield, UK), and CoolSnapHQ High Speed Monochrome CCD camera (Photometrics, Marlow, UK) run by Metamorph image acquisition software. Image stacks $(0.2 \mu \mathrm{m}$ steps $)$ were deconvolved using AutoquantX (Media Cybernetics, UK). Micrographs were saved from source programs as 12-bit.tif files and analyzed with Image Pro Plus software and/or prepared for figures using Photoshop 8.0.

The positional distribution of the lacO array was determined using a macro (available on request) written in Visual Basic within Image Pro Plus. In brief, the total nuclear area was automatically measured on DAPI images, then divided into five shells of equal area through eroding $20 \%$ of total area from the outer limits of the DAPI-defined nucleus. The nuclear shell containing the lacO spot was determined, exported to Microsoft Excel and summed for each cell. The same shells were also applied for DAPI or chromosome intensity measurements where a manually intensity-thresholded and background subtracted image of the chromosomes was used (two-dimensional shell erosion macro).

To quantify the three-dimensional position of FISHlabeled chromosomes within the nucleus, a macro (threedimensional shell erosion macro) was devised within Image Pro Plus 7.0 software based on a similar erosion script to that used for the two-dimensional analysis. Prior to running the macro, images of Alexa 488-labeled chromosomes and DAPI-labeled nuclei were deconvolved using Autoquant $\mathrm{X}$, exported into Image Pro Plus 7.0, and saved as two separate files.

Within the macro each $\mathrm{z}$ level of the nucleus was automatically thresholded and converted into a threedimensional binary image so that weaker DAPI stained regions such as nucleoli would not be interpreted as holes for nuclear area when applying the erosion script. The chromosomes were manually thresholded and then converted into a three-dimensional binary image. This binary image required a minimum density so that background spots would be eliminated. Though this had the disadvantage that some control cells with very wide and diffuse chromosome distributions in the nuclear interior had reduced internal chromosome measurements, nonetheless, the only effect on data interpretation was to lessen differences between the controls and NETs that repositioned chromosomes. A three-dimensional distance filter was applied to the three-dimensional nucleus image to allow erosion of the nucleus based on distance from the center. Three-dimensional binary images were produced from the distance image by dividing the total area by 6 and then eroding the area by $1 / 6$ th each time from the preceding image to produce six images.

The binary chromosome image was then combined with each of the six eroding nucleus images and the degree of co-localization was then measured in each combined image and the percentage of chromosome in each eroded three-dimensional nuclear area calculated. Subtraction of the percentage in sequential shells then gave the percentage of the chromosome in each unique shell.

To quantify nuclear size, the area of the midplane was measured using Image Pro Plus 7.0 and plotted as distribution plots. For nuclear shape the longest and shortest distances across the midplane images were also measured automatically with the built-in functions of the software and plotted as scatter plots.

\section{Bioinformatics analysis and statistics}

Repositioning of lacO arrays was assessed by comparing the number of arrays present within the outermost shell (shell 1) against the internal area (combined shells $3+4$ +5 ) between each NET and the MRFP control by means of the one-tailed chi-squared test with a significance threshold of $P<0.01$ for high stringency or $P<0.05$ for low stringency.

Chromosomes occupy a large area, compared to the lacO arrays, spanning several shells. For this reason, the chromosome data were calculated as the percentage of the total chromosome signal intensity that was contained within each shell. Chromosome repositioning was assessed by comparing the distribution of chromosome 
intensities within the periphery (shells 1 and 2) between each NET and the NLS-GFP, scramble siRNA and NET siRNA or liver and kidney by means of the KS test with a significance threshold of $P<0.01$ for high stringency and $P<0.05$ for low stringency.

\section{Additional material}

Additional file 1: Table S1 - statistics for Figures 1, 2, 5, 6, 7, and 9 . Repositioning of lacO arrays (for Figures $1 \mathrm{~d}$ and $2 \mathrm{~b}, \mathrm{c}$ ) was assessed by comparing the number of arrays present within the outermost shell (shell 1) against the internal area (combined shells $3+4+5$ ) between each NET and the mRFP control by means of the one-tailed chi-squared test. Chromosome repositioning was assessed by comparing the distribution of chromosome intensities within the periphery (combined shells 1 and 2) between each NET and the NLS-GFP (Figures $5 b$ and $6 c$ ), scramble siRNA and NET siRNA (Figures $7 \mathrm{~b}$ and $9 \mathrm{e}, \mathrm{f}$ ) or liver and kidney (Figure $9 b)$ by means of the KS test. $P$-values for each sample are listed with a significance threshold of $P<0.01$ for high stringency (red) or $P<0.05$ for low stringency (blue).

Additional file 2: Table S2. Quantification of bands in western blots was performed using the LI-COR Odyssey Application Software version 3.0. Integrated intensities were calculated from an equal size box drawn around each band in each tissue (Table S2a). To assess the variation of protein abundance among tissues, the intensities were summed within each protein for all tissues, and then expressed as a percentage of the signal per tissue (Table S2b).

\section{Abbreviations}

BSA: bovine serum albumin; FISH: fluorescence in situ hybridization; GFP: green fluorescent protein; KS: Kolmogorov-Smirnov; lacO: lac operator; LBR: lamin B receptor; mRFP: monomeric red fluorescent protein; NE: nuclear envelope; NET: nuclear envelope transmembrane protein; NLS: nuclear localization signal; PBS: phosphate-buffered saline; siRNA: small interfering RNA.

\section{Authors' contributions}

NZ designed experiments, performed most of the experimentation and data analysis, and helped draft the manuscript. SB trained NZ in FISH techniques and helped with human tissue section staining. DAK wrote the chromosome repositioning analysis algorithms and helped with data analysis. JIH helped with data analysis. VL did preliminary work on the lacO array repositioning screen. NK helped to immunostain human tissue blots. DGB helped with protein quantification. KNR and GEM generated the NET45 antibody. DJH provided human tissue sections. WAB provided reagents and helped write the manuscript. ECS designed the study and wrote the manuscript. All authors read and approved the final version of the manuscript.

\section{Acknowledgements}

We thank Rita Roberti and Paola Vagnarelli for the TM7SF2/NET47 and the nucleolin antibodies, Jonathan Chubb and Paul Perry for cell lines and the template for the original erosion script, Martin Waterfall for FACS assistance, and Michael Robson for critical reading of the manuscript. NZ was supported in part by a University of Edinburgh Staff Scholarship, KNR by a studentship from the Higher Education Funding Council (Wales) and DGB by the Darwin Trust of Edinburgh. The Muscular Dystrophy Association (USA) supports monoclonal antibody development in the laboratory of GEM. WAB was supported by an ERC Advanced grant (ERC-2009-AdG-20090506) and by the Medical Research Council, UK. This work was principally supported by Wellcome Trust Senior Research Fellowship (076616 and 095209) to ECS and Wellcome Trust Centre for Cell Biology core funding 077707.

\section{Author details}

${ }^{1}$ The Wellcome Trust Centre for Cell Biology, University of Edinburgh, Mayfield Road, Edinburgh, EH9 3JR, UK. ${ }^{2}$ MRC Human Genetics Unit, Institute of Genetics and Molecular Medicine, Crewe Road, Edinburgh, EH4 2XU, UK.
${ }^{3}$ Wolfson Centre for Inherited Neuromuscular Disease, RJAH Orthopaedic Hospital, Twympath Lane, Oswestry, SY10 7AG, UK. ${ }^{4}$ Institute for Science and Technology in Medicine, Keele University, Keele, Staffordshire, ST4 7QB, UK.

${ }^{5}$ School of Medicine, Medical andBiological Sciences, University of St

Andrews, North Haugh, St Andrews, KY16 9TF, UK.

Received: 20 September 2012 Revised: 11 February 2013

Accepted: 15 February 2013 Published: 15 February 2013

\section{References}

1. Bolzer A, Kreth G, Solovei I, Koehler D, Saracoglu K, Fauth C, Muller S, Eils R, Cremer C, Speicher MR, Cremer T: Three-dimensional maps of all chromosomes in human male fibroblast nuclei and prometaphase rosettes. PLoS Biol 2005, 3:e157.

2. Boyle S, Gilchrist S, Bridger JM, Mahy NL, Ellis JA, Bickmore WA: The spatial organization of human chromosomes within the nuclei of normal and emerin-mutant cells. Hum Mol Genet 2001, 10:211-219.

3. Croft JA, Bridger JM, Boyle S, Perry P, Teague P, Bickmore WA: Differences in the localization and morphology of chromosomes in the human nucleus. J Cell Biol 1999, 145:1119-1131.

4. Guelen L, Pagie L, Brasset E, Meuleman W, Faza MB, Talhout W, Eussen BH, de Klein A, Wessels L, de Laat W, van Steensel B: Domain organization of human chromosomes revealed by mapping of nuclear lamina interactions. Nature 2008, 453:948-951.

5. Wiblin AE, Cui W, Clark AJ, Bickmore WA: Distinctive nuclear organisation of centromeres and regions involved in pluripotency in human embryonic stem cells. J Cell Sci 2005, 118:3861-3868.

6. Bridger JM, Boyle S, Kill IR, Bickmore WA: Re-modelling of nuclear architecture in quiescent and senescent human fibroblasts. Curr Biol 2000, 10:149-152.

7. Mehta IS, Amira M, Harvey AJ, Bridger JM: Rapid chromosome territory relocation by nuclear motor activity in response to serum removal in primary human fibroblasts. Genome Biol 2010, 11:R5.

8. Mehta IS, Eskiw CH, Arican HD, Kill IR, Bridger JM: Farnesyltransferase inhibitor treatment restores chromosome territory positions and active chromosome dynamics in Hutchinson-Gilford progeria syndrome cells. Genome Biol 2011, 12:R74.

9. Kosak ST, Scalzo D, Alworth SV, Li F, Palmer S, Enver T, Lee JS, Groudine M: Coordinate gene regulation during hematopoiesis is related to genomic organization. PLoS Biol 2007, 5:e309.

10. Meister $P$, Towbin BD, Pike BL, Ponti A, Gasser SM: The spatial dynamics of tissue-specific promoters during C. elegans development. Genes Dev 2010, 24:766-782.

11. Parada LA, McQueen PG, Munson PJ, Misteli T: Conservation of relative chromosome positioning in normal and cancer cells. Curr Biol 2002, 12:1692-1697.

12. Parada $L A$, McQueen $P G$, Misteli T: Tissue-specific spatial organization of genomes. Genome Biol 2004, 5:R44.

13. Solovei I, Kreysing M, Lanctot C, Kosem S, Peichl L, Cremer T, Guck J, Joffe B: Nuclear architecture of rod photoreceptor cells adapts to vision in mammalian evolution. Cell 2009, 137:356-368.

14. Peric-Hupkes D, Meuleman W, Pagie L, Bruggeman SW, Solovei I, Brugman W, Graf S, Flicek P, Kerkhoven RM, van Lohuizen M, Reinders M, Wessels L, van Steensel B: Molecular maps of the reorganization of genome-nuclear lamina interactions during differentiation. Mol Cell 2010, 38:603-613.

15. Anderson DJ, Vargas JD, Hsiao JP, Hetzer MW: Recruitment of functionally distinct membrane proteins to chromatin mediates nuclear envelope formation in vivo. J Cell Biol 2009, 186:183-191.

16. Ulbert S, Antonin W, Platani M, Mattaj IW: The inner nuclear membrane protein Lem2 is critical for normal nuclear envelope morphology. FEBS Lett 2006, 580:6435-6441.

17. Finlan LE, Sproul D, Thomson I, Boyle S, Kerr E, Perry P, YIstra B, Chubb JR, Bickmore WA: Recruitment to the nuclear periphery can alter expression of genes in human cells. PLoS Genet 2008, 4:e1000039.

18. Kumaran Rl, Spector DL: A genetic locus targeted to the nuclear periphery in living cells maintains its transcriptional competence. $J$ Cell Biol 2008, 180:51-65.

19. Reddy KL, Zullo JM, Bertolino E, Singh H: Transcriptional repression mediated by repositioning of genes to the nuclear lamina. Nature 2008 , 452:243-247. 
20. Mattout-Drubezki A, Gruenbaum Y: Dynamic interactions of nuclear lamina proteins with chromatin and transcriptional machinery. Cell Mol Life Sci 2003, 60:2053-2063.

21. Brown CR, Kennedy CJ, Delmar VA, Forbes DJ, Silver PA: Global histone acetylation induces functional genomic reorganization at mammalian nuclear pore complexes. Genes Dev 2008, 22:627-639.

22. Capelson M, Liang Y, Schulte R, Mair W, Wagner U, Hetzer MW: Chromatinbound nuclear pore components regulate gene expression in higher eukaryotes. Cell 2010, 140:372-383.

23. Kalverda B, Pickersgill H, Shloma W, Fornerod M: Nucleoporins directly stimulate expression of developmental and cell-cycle genes inside the nucleoplasm. Cell 2010, 140:360-371.

24. Makatsori D, Kourmouli N, Polioudaki H, Shultz LD, McLean K, Theodoropoulos PA, Singh PB, Georgatos SD: The inner nuclear membrane protein lamin $B$ receptor forms distinct microdomains and links epigenetically marked chromatin to the nuclear envelope. J Biol Chem 2004, 279:25567-25573

25. Pickersgill $H$, Kalverda B, de Wit E, Talhout W, Fornerod M, van Steensel B: Characterization of the Drosophila melanogaster genome at the nuclear lamina. Nat Genet 2006, 38:1005-1014.

26. Malhas A, Lee CF, Sanders R, Saunders NJ, Vaux DJ: Defects in lamin B1 expression or processing affect interphase chromosome position and gene expression. J Cell Biol 2007, 176:593-603.

27. Zullo JM, Demarco IA, Pique-Regi R, Gaffney DJ, Epstein CB, Spooner CJ, Luperchio TR, Bernstein BE, Pritchard JK, Reddy KL, Singh H: DNA sequence-dependent compartmentalization and silencing of chromatin at the nuclear lamina. Cell 2012, 149:1474-1487.

28. Mewborn SK, Puckelwartz MJ, Abuisneineh F, Fahrenbach JP, Zhang Y, MacLeod H, Dellefave L, Pytel P, Selig S, Labno CM, Reddy K, Singh H, McNally E: Altered chromosomal positioning, compaction, and gene expression with a lamin A/C gene mutation. PLoS One 2010, 5:e14342.

29. Schirmer EC, Florens L, Guan T, Yates JRr, Gerace L: Nuclear membrane proteins with potential disease links found by subtractive proteomics. Science 2003, 301:1380-1382.

30. Malik P, Korfali N, Srsen V, Lazou V, Batrakou DG, Zuleger N, Kavanagh DM, Wilkie GS, Goldberg MW, Schirmer EC: Cell-specific and lamin-dependent targeting of novel transmembrane proteins in the nuclear envelope. Cell Mol Life Sci 2010, 67:1353-1369.

31. Zuleger N, Kelly DA, Richardson AC, Kerr AR, Goldberg MW, Goryachev AB, Schirmer EC: System analysis shows distinct mechanisms and common principles of nuclear envelope protein dynamics. J Cell Biol 2011, 193:109-123.

32. Chubb JR, Boyle S, Perry P, Bickmore WA: Chromatin motion is constrained by association with nuclear compartments in human cells. Curr Biol 2002, 12:439-445.

33. Rajapakse I, Groudine M, Mesbahi M: Dynamics and control of statedependent networks for probing genomic organization. Proc Natl Acad Sci USA 2011, 108:17257-17262.

34. Malik P, Tabarraei A, Kehlenbach RH, Korfali N, Iwasawa R, Graham SV, Schirmer EC: Herpes simplex virus ICP27 protein directly interacts with the nuclear pore complex through Nup62, inhibiting host nucleocytoplasmic transport pathways. J Biol Chem 2012, 287:12277-12292.

35. Kalhor R, Tjong H, Jayathilaka N, Alber F, Chen L: Genome architectures revealed by tethered chromosome conformation capture and population-based modeling. Nat Biotechnol 2012, 30:90-98.

36. Albiez H, Cremer M, Tiberi C, Vecchio L, Schermelleh L, Dittrich S, Kupper K, Joffe B, Thormeyer T, von Hase J, Yang S, Rohr K, Leonhardt H, Solovei I, Cremer C, Fakan S, Cremer T: Chromatin domains and the interchromatin compartment form structurally defined and functionally interacting nuclear networks. Chromosome Res 2006, 14:707-733.

37. Ragoczy T, Telling A, Sawado T, Groudine M, Kosak ST: A genetic analysis of chromosome territory looping: diverse roles for distal regulatory elements. Chromosome Res 2003, 11:513-525.

38. Zink D, Amaral MD, Englmann A, Lang S, Clarke LA, Rudolph C, Alt F, Luther K, Braz C, Sadoni N, Rosenecker J, Schindelhauer D: Transcriptiondependent spatial arrangements of CFTR and adjacent genes in human cell nuclei. J Cell Biol 2004, 166:815-825.

39. Soullam B, Worman HJ: The amino-terminal domain of the lamin B receptor is a nuclear envelope targeting signal. J Cell Biol 1993, 120:1093-1100.
40. Kim SH, McQueen PG, Lichtman MK, Shevach EM, Parada LA, Misteli T: Spatial genome organization during T-cell differentiation. Cytogenet Genome Res 2004, 105:292-301.

41. Stadler S, Schnapp V, Mayer R, Stein S, Cremer C, Bonifer C, Cremer T, Dietzel S: The architecture of chicken chromosome territories changes during differentiation. BMC Cell Biol 2004, 5:44.

42. Aden DP, Fogel A, Plotkin S, Damjanov I, Knowles BB: Controlled synthesis of HBsAg in a differentiated human liver carcinoma-derived cell line. Nature 1979, 282:615-616.

43. Solovei I, Wang AS, Thanisch K, Schmidt CS, Krebs S, Zwerger M, Cohen TV, Devys D, Foisner R, Peichl L, Herrmann H, Blum H, Engelkamp D, Stewart $C L$, Leonhardt $H$, Joffe B: LBR and Lamin A/C sequentially tether peripheral heterochromatin and inversely regulate differentiation. Cell 2013, 152:584-598

44. Towbin BD, Gonzalez-Aguilera C, Sack R, Gaidatzis D, Kalck V, Meister P, Askjaer P, Gasser SM: Step-wise methylation of histone H3K9 positions heterochromatin at the nuclear periphery. Cell 2012, 150:934-947.

45. Goldberg M, Harel A, Brandeis M, Rechsteiner T, Richmond TJ, Weiss AM, Gruenbaum Y: The tail domain of lamin Dm0 binds histones $\mathrm{H} 2 \mathrm{~A}$ and H2B. Proc Natl Acad Sci USA 1999, 96:2852-2857.

46. Hoger TH, Krohne $G$, Kleinschmidt JA: Interaction of Xenopus lamins A and LII with chromatin in vitro mediated by a sequence element in the carboxyterminal domain. Exp Cell Res 1991, 197:280-289.

47. Taniura H, Glass C, Gerace L: A chromatin binding site in the tail domain of nuclear lamins that interacts with core histones. J Cell Biol 1995, 131:33-44

48. Mattout A, Pike BL, Towbin BD, Bank EM, Gonzalez-Sandoval A, Stadler MB, Meister P, Gruenbaum Y, Gasser SM: An EDMD mutation in C. elegans lamin blocks muscle-specific gene relocation and compromises muscle integrity. Curr Biol 2011, 21:1603-1614

49. Meaburn KJ, Cabuy E, Bonne G, Levy N, Morris GE, Novelli G, Kill IR, Bridger JM: Primary laminopathy fibroblasts display altered genome organization and apoptosis. Aging Cell 2007, 6:139-153.

50. Gudise S, Figueroa RA, Lindberg R, Larsson V, Hallberg E: Samp1 is functionally associated with the LINC complex and A-type lamina networks. J Cell Sci 2011, 124:2077-2085.

51. King MC, Drivas TG, Blobel G: A network of nuclear envelope membrane proteins linking centromeres to microtubules. Cell 2008, 134:427-438.

52. Holmer L, Pezhman A, Worman HJ: The human lamin B receptor/sterol reductase multigene family. Genomics 1998, 54:469-476.

53. Roberti R, Bennati AM, Galli G, Caruso D, Maras B, Aisa C, Beccari T, Della Fazia MA, Servillo G: Cloning and expression of sterol Delta 14-reductase from bovine liver. Eur J Biochem 2002, 269:283-290.

54. Silve S, Dupuy PH, Ferrara P, Loison G: Human lamin B receptor exhibits sterol C14-reductase activity in Saccharomyces cerevisiae. Biochim Biophys Acta 1998, 1392:233-244.

55. Worman HJ, Lazaridis I, Georgatos SD: Nuclear lamina heterogeneity in mammalian cells. Differential expression of the major lamins and variations in lamin B phosphorylation. J Biol Chem 1988, 263:12135-12141.

56. Ye Q, Worman $\mathrm{HJ}$ : Interaction between an integral protein of the nuclear envelope inner membrane and human chromodomain proteins homologous to Drosophila HP1. J Biol Chem 1996, 271:14653-14656.

57. Ye Q, Callebaut I, Pezhman A, Courvalin JC, Worman HJ: Domain-specific interactions of human HP1-type chromodomain proteins and inner nuclear membrane protein LBR. J Biol Chem 1997, 272:14983-14989.

58. Clowney EJ, LeGros MA, Mosley CP, Clowney FG, MarkenskoffPapadimitriou EC, Myllys M, Barnea G, Larabell CA, Lomvardas S: Nuclear aggregation of olfactory receptor genes governs their monogenic expression. Cell 2012, 151:724-737.

59. Chen $I H$, Huber M, Guan T, Bubeck A, Gerace L: Nuclear envelope transmembrane proteins (NETs) that are up-regulated during myogenesis. BMC Cell Biol 2006, 7:38.

60. Liu GH, Guan T, Datta K, Coppinger J, Yates J, Gerace L: Regulation of myoblast differentiation by the nuclear envelope protein NET39. Mol Cell Biol 2009, 29:5800-5812.

61. Bennati AM, Schiavoni G, Franken S, Piobbico D, Della Fazia MA, Caruso D, De Fabiani E, Benedetti L, Cusella De Angelis MG, Gieselmann V, Servillo G, Beccari T, Roberti R: Disruption of the gene encoding 3beta-hydroxysterol Delta-reductase (Tm7sf2) in mice does not impair cholesterol biosynthesis. FEBS J 2008, 275:5034-5047. 
62. Hutten S, Flotho A, Melchior F, Kehlenbach RH: The Nup358-RanGAP complex is required for efficient importin alpha/beta-dependent nuclear import. Mol Biol Cell 2008, 19:2300-2310.

63. Man N, Morris GE: A rapid method for generating large numbers of highaffinity monoclonal antibodies from a single mouse. In The Protein Protocols Handbook.. 3 edition. Edited by: Walker J. Totowa, NJ: Humana Press; 2009:1961-1974.

64. Schirmer EC, Guan T, Gerace L: Involvement of the lamin rod domain in heterotypic lamin interactions important for nuclear organization. J Cell Biol 2001, 153:479-489.

65. Manilal S, Nguyen TM, Sewry CA, Morris GE: The Emery-Dreifuss muscular dystrophy protein, emerin, is a nuclear membrane protein. Hum Mol Genet 1996, 5:801-808.

66. Cremer M, Grasser F, Lanctot C, Muller S, Neusser M, Zinner R, Solovei I, Cremer T: Multicolor 3D fluorescence in situ hybridization for imaging interphase chromosomes. Methods Mol Biol 2008, 463:205-239.

doi:10.1186/gb-2013-14-2-r14

Cite this article as: Zuleger et al: Specific nuclear envelope

transmembrane proteins can promote the location of chromosomes to and from the nuclear periphery. Genome Biology 2013 14:R14.

\section{Submit your next manuscript to BioMed Central} and take full advantage of:

- Convenient online submission

- Thorough peer review

- No space constraints or color figure charges

- Immediate publication on acceptance

- Inclusion in PubMed, CAS, Scopus and Google Scholar

- Research which is freely available for redistribution

Submit your manuscript at www.biomedcentral.com/submit 